\title{
Acceleration of Non-Equidiffusive Flames in Channels: Computational Simulations and Analytical Studies
}

\author{
Serdar A. Bilgili
}

Follow this and additional works at: https://researchrepository.wvu.edu/etd

\section{Recommended Citation}

Bilgili, Serdar A., "Acceleration of Non-Equidiffusive Flames in Channels: Computational Simulations and Analytical Studies" (2015). Graduate Theses, Dissertations, and Problem Reports. 5209.

https://researchrepository.wvu.edu/etd/5209

This Thesis is protected by copyright and/or related rights. It has been brought to you by the The Research Repository @ WVU with permission from the rights-holder(s). You are free to use this Thesis in any way that is permitted by the copyright and related rights legislation that applies to your use. For other uses you must obtain permission from the rights-holder(s) directly, unless additional rights are indicated by a Creative Commons license in the record and/ or on the work itself. This Thesis has been accepted for inclusion in WVU Graduate Theses, Dissertations, and Problem Reports collection by an authorized administrator of The Research Repository @ WVU. For more information, please contact researchrepository@mail.wvu.edu. 
Acceleration of Non-Equidiffusive Flames in Channels:

Computational Simulations and Analytical Studies

\author{
Serdar A. Bilgili
}

Thesis submitted to the

Benjamin M. Statler College of Engineering and Mineral Resources

at West Virginia University

in partial fulfillment of the requirements for the degree of

Master of Science

in

Mechanical Engineering

V'yacheslav Akkerman, Ph.D., Chair

Ismail B. Celik, Ph.D.

Cosmin Dumitrescu, Ph.D.

Damir Valiev, Ph.D.

Department of Mechanical and Aerospace Engineering

Morgantown, West Virginia

2015

Keywords: Finite flame thickness, flame stretch, (non) equidiffusive flames, Markstein and Lewis numbers, combustion instability, flame acceleration, wall friction, flame thickening and channeling, computational simulation, analytical formulation.

Copyright 2015 Serdar Ali Bilgili 


\title{
ABSTRACT \\ Acceleration of Non-Equidiffusive Flames in Channels: Computational Simulations and Analytical Studies
}

\author{
Serdar A. Bilgili
}

When a premixed flame front spreads in a narrow pipe, wall friction continuously distorts the flame shape. As a result, the flame front acquires a larger surface area, consumes more fuel per unit time and, thereby, propagates faster. While this mechanism of flame acceleration due to wall friction has widely been studied, especially within the last decade, the analytical and computational studies were mostly devoted to equidiffusive flames, where the Lewis number, defined as the thermal to mass diffusivity ratio, is unity, $L e=1$. However, in reality thermal and mass diffusion are typically not balanced, especially in rich and lean mixtures. Hence, the microscale, diffusional-thermal effects may appear comparable with macro-scale phenomena such as wall friction. The present work sheds the light on the dynamics and morphology of $L e \neq 1$ flames in channels. Specifically, it studies, by means of computational and analytical endeavors, how the interplay of finite flame thickness, stretch effect and the thermal-molecular diffusion influence the overall flame acceleration scenario. It is shown that $L e>1$ flames accelerate slower, due to an effective thickening of the flame front. In contrast, $L e<1$ flames exhibit faster acceleration due to effective flame channeling and other morphological deformations resembling the diffusional-thermal (DT) instability. The analysis also incorporates the internal transport flame properties into the theory of flame acceleration due to wall friction, by means of the Markstein number, $M k$, that characterizes the flame response to curvature and stretch. Being a positive or negative function of thermal-chemical combustion parameters, such as the thermal expansion ratio and the Lewis and Zel'dovich numbers, the Markstein number either restrains or promotes the flame acceleration. While $M k$ may substantially facilitate the flame acceleration in narrow channels, this effects diminishes with the increase in the channel width. The analysis is accompanied by extensive numerical simulations of the Navier-Stokes and combustion equations, which clarify the impact of the Lewis number on the flame acceleration. It is obtained that, for $L e$ lower than a certain critical value, at the initial stage of flame acceleration, globallyconvex flame fronts split into two or more "fingers", accompanied by a drastic increase in the flame surface area and associated enhancement of the flame acceleration. Later, however, the flame fingers meet, promptly consuming the troughs, which rapidly diminishes the flame surface

area and moderates the acceleration. Eventually, this results in a single, globally-convex flame front that keeps accelerating. Overall, the thermal-diffusive effects facilitate the flame acceleration scenario, thereby advancing a potential deflagration-to-detonation transition. 


\section{Acknowledgements}

A big part of this thesis would not come into being if it were not for several key individuals, to which I would like to extend my humble gratitude and sincere appreciation.

Primarily, I would like to thank Dr. V'yacheslav Akkerman, who was the initiator of my career in the MAE department, and the WVU family. Without his guidance, patience, and full support, I would not have done nearly as much as I have accomplished so far. I also would like to extend my special thanks to the committee members for their precious time: I am grateful to Dr. Ismail B. Celik, who has been a valuable mentor for me even beyond academics, and Dr. Cosmin Dumitrescu for his gracious support.

Furthermore, I do owe my deepest and sincere thanks to Drs. Vitaly Bychkov and Damir Valiev of Umea University in Sweden, for their everlasting cooperation and consulting. Without Dr. Bychkov's amazing expertise on the combustion physics, and Dr. Valiev's excellent computational skills, our research group would unequivocally lack a great support.

I would not even be writing these acknowledgements without the countless useful discussions and support of my friends and colleagues in the ACCORD (Analytical and Computational Combustion Research Directions) group, CFD\&AMP (Computational Fluid Dynamics and Applied Multi-Physics Center), and CAFEE (Center for Alternative Fuels, Engines, and Emissions). In this regard, my special thanks go to Sinan Demir, Berk Demirgok, Hayri Sezer, and Orlando Ugarte who have substantially assisted my computational endeavors. I also express my sincere thanks to WVU HPC (High Performance Computing) facility and its staff for their invaluable effort to run everything flawless.

Finally, I would like to dedicate my last words to all my family members, who have provided me nothing but love, respect, support and companionship, hence making me the luckiest individual. 
I dedicate this work as any other product of mine to my beloved Merve, without whose support and companionship I could not have accomplished so far. 


\section{Table of Contents}

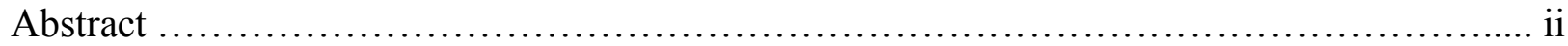

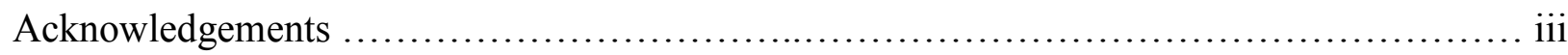

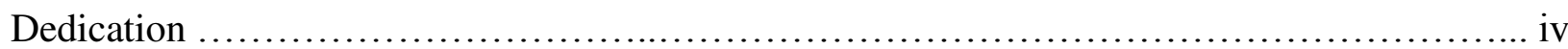



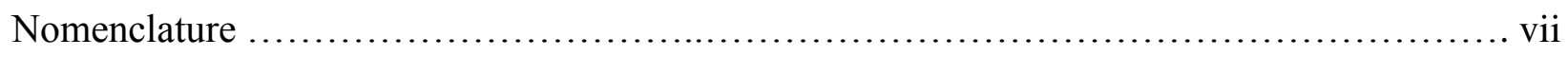

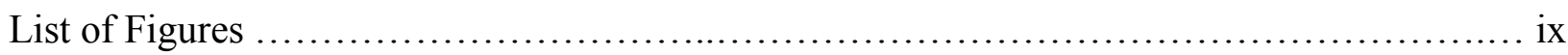

List of Tables .............................................................................

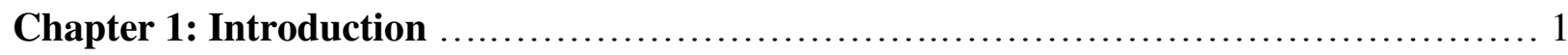

1.1. Motivation and Literature on the Flame Acceleration Mechanisms ................. 1

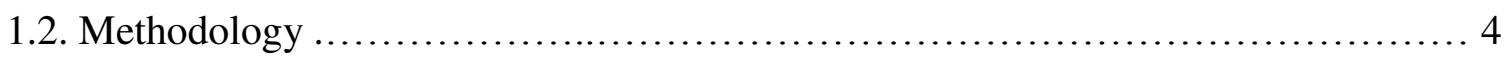

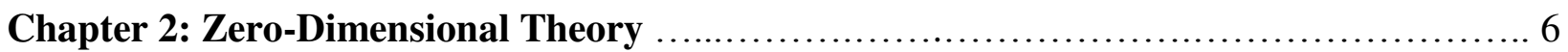

2.1. Critical Parameters of the Diffusional-Thermal (DT) Instability .................. 6

2.2. Neutral Curves ................................................................. 7

Chapter 3: Description of the Numerical Approach .................................. 10

3.1. Basic Equations ............................................................ 10

3.2. Numerical Scheme ....................................................... 11

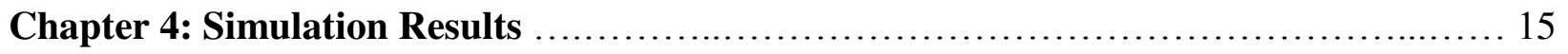

4.1. Equidiffusive Flames....................................................... 15

4.2. Non-Equidiffusive Flames ...................................................... 19

4.2.1. $L e>1$ results ..................................................... 19

4.2.2. $L e<1$ results ....................................................... 23 


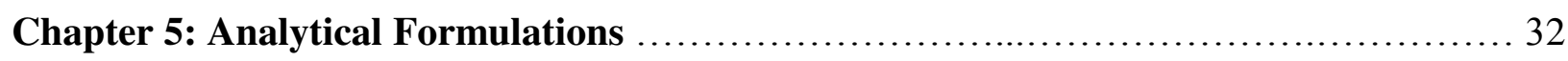

5.1. Self-Similar Consideration ................................................... 32

5.2. Alternative Formulation ................................................... 40

5.2.1. Plane parallel flow and notation correction ........................... 41

5.2.2. Flame function sign correction .......................................... 41

5.2.3. Reference frame correction ...................................... 42

5.2.4. Curvature term correction ........................................ 42

5.2.5. Solution for the modified flame evolution equation .....................43

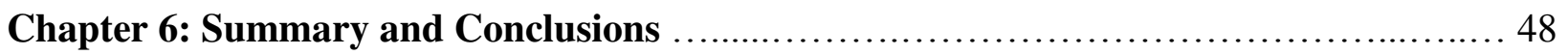

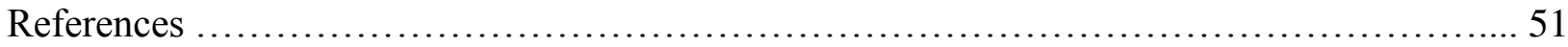




\section{Nomenclature}

\section{Latin}

\begin{tabular}{cc}
$A_{w}$ & Scaled flame surface area \\
$C_{P}$ & Constant pressure heat capacity \\
$C_{V}$ & Constant volume heat capacity \\
$D_{f}$ & Length of a 2D flame front \\
$D_{t h}$ & Thermal diffusion coefficient \\
$D L$ & Darrieus-Landau \\
$D T$ & Diffusional-thermal \\
$D D T$ & Deflagration-to-detonation transition \\
$E_{A}$ & Reaction activation energy \\
$E_{A, C}$ & Critical activation energy \\
$f$ & Flame shape function \\
$H$ & Enthalpy \\
$h$ & Temperature dependence function of transport \\
$L_{f}$ & coefficients \\
$L e$ & Flame thickness \\
$L e_{C}$ & Lewis number \\
$m$ & Critical Lewis number \\
$M a$ & Species molecular weight \\
$M k$ & Mach number \\
$M k_{e f f}$ & Markstein number \\
$M k_{C}$ & Effective Markstein number \\
$N$ & Critical Markstein number \\
$\widehat{\boldsymbol{n}}$ & Scaled increase in flame surface area \\
$P$ & Surface normal vector \\
$p$ & Pressure \\
$P_{f}$ & Scaled pressure \\
$P r$ & Fuel pressure \\
$Q$ & Prandtl number \\
$q_{j}$ & Reaction energy release \\
$R$ & Energy diffusion vector \\
$R_{u}$ & Channel radius (half-width) \\
$R e$ & Tempersal gas constant \\
$R e_{f l o w}$ & Reynolds number \\
$S c$ & \\
$T$ & \\
& Schmidt number \\
\hline &
\end{tabular}




$$
\begin{gathered}
T_{b} \\
T_{f} \\
\boldsymbol{u} \\
U_{f} \\
U_{w} \\
u_{z} \\
\boldsymbol{w} \\
w_{z} \\
\boldsymbol{v} \\
Y \\
\mathrm{Ze} \\
\mathrm{Ze} C
\end{gathered}
$$

\section{Greek}

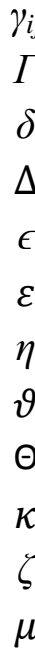

Burnt matter temperature

Fuel temperature Flow velocity field Laminar flame velocity Total burning rate Flow velocity z-component Scaled flow velocity field Scaled flow velocity z-component Scaled transverse velocity field Fuel mixture mass fraction Zel'dovich number Critical Zel'dovich number

The stress tensor Phenomenological constant Scaled flame thickness Infinitesimal flame surface area

Correction parameter Internal energy Scaled x-coordinate Scaled temperature Thermal expansion coefficient Curvature Dynamic viscosity Square root of acceleration rate multiplied with Reynolds number

Kinematic viscosity Scaled z-coordinate Local flame shape function Density

Scaled flame acceleration rate Zeroth-order acceleration rate First-order correction for acceleration rate Scaled time, $t U_{f} / R$

Reaction time constant Spatial component of the flame shape function Zeroth-order flame shape function First-order correction for flame shape function The Del (nabla) operator 


\section{List of Figures}

Figure 1.1: A schematic of wall-friction (a.k.a. Shelkin) mechanism, yielding an exponential acceleration regime.

Figure 1.2: Evolution of the flame isotherms, from 600 to $2100 \mathrm{~K}$ with the step of $300 \mathrm{~K}$, in the simulation run for $R e=25$, and $\operatorname{Pr}=1$. [17]

Figure 2.1: $Z e_{C}$ as a function of $\Theta$ for $h(\vartheta)=1$ (dashed), and $h(\vartheta)=\vartheta^{1 / 2}$ (solid). Markers correspond to current simulation parameters.

Figure 2.2: $\quad L e_{C}$ versus $\Theta$ for fixed $Z e=3.5 ; 10 ; 20 ; 50$. Solid lines use $h(\theta)=1$, and dashed lines use $h(\vartheta)=\vartheta^{1 / 2}$.

Figure 2.3: $L e_{C}$ versus $Z e$ for fixed $\Theta=[3,5,8,12]$. Solid lines use $h(\theta)=1$, and dashed lines use $h(\vartheta)=\vartheta^{1 / 2}$.

Figure 3.1: A sketch of the adaptive non-uniform grid with variable resolution [46].

Figure 3.2: A resolution test for the flame tip position versus time, where different mesh sizes are considered which are scaled with respect to the flame thickness [46]. $L e=1$.

Figure 3.3: A resolution test for the flame tip position versus time, similar to Fig. (3.2), but for the current simulations. $L e=0.2$.

Figure 4.1: Color temperature snapshots for flame propagation in channels with $L e=1$, $R e=10$.

Figure 4.2: Color temperature snapshots for flame propagation in channels with $L e=1$, $R e=20$.

Figure 4.3: The scaled flame tip velocity, $U_{t i p} / U_{f}$, the flame surface area, $A_{w} / D$, and the scaled total burning rate, $U_{w} / U_{f}$, versus the scaled time for $L e=1$ and $R e=5-35$.

Figure 4.4: The scaled burning rate versus the scaled time for $L e=1$ on semi-log plot, along with the exponential regression fits.

Figure 4.5: The scaled exponential flame acceleration rate versus the scaled time for $L e=1$.

Figure 4.6: Color temperature snapshots for flame propagation in channels with $L e=2$, $R e=10$.

Figure 4.7: Color temperature snapshots for flame propagation in channels with $L e=2$, $R e=20$. 
Figure 4.8: The scaled flame tip velocity, $U_{t i p} / U_{f}$, the flame surface area, $A_{w} / D$, and the scaled total burning rate, $U_{w} / U_{f}$, versus the scaled time for $\mathrm{Le}=2.0$ and $R e=10,20$.

Figure 4.9: The scaled burning rate versus the scaled time for $L e=2$, along with exponential regression fits.

Figure 4.10: The scaled exponential flame acceleration rate versus the scaled time for $L e=2$.

Figure 4.11: Evolution of the central trough for non-equidiffusive flames $(R e=25, L e=0.2)$.

Figure 4.12: Evolution of the central trough for non-equidiffusive flames $(R e=20, L e=0.2)$.

Figure 4.13: The scaled flame tip position $(z / R)$ versus the scaled time $\left(\tau=t U_{f} / R\right)$, for the fixed $R e=10\left(R=20 \mathrm{~L}_{\mathrm{f}}\right)$ and various $L e$ values.

Figure 4.14: Evolution of the "stages" of non-equidiffusive flames $(R e=15, L e=0.2)$.

Figure 4.15: The scaled tip velocity versus the scaled time $\left(\tau=t U_{f} / R\right)$ for fixed $R e=20$ $\left(R=40 L_{f}\right)$ and various $L e$.

Figure 4.16: The scaled burning rate $\left(U_{w} / U_{f}\right)$ versus the scaled time $\left(\tau=t U_{f} / R\right)$, for the fixed $L e=0.2$, and various $R e=10 \sim 35$.

Figure 4.17: $L e_{C}$ versus $R e$ (see Table 1), where the exponential regression model is fitted with the coefficient of determination value of $R^{2}=0.998$.

Figure 4.18: a) Evolution of the trough instability for the case when it is suppressed, i.e. $L e>L e_{C}(R e=10, L e=0.6)$, b) Evolution of the trough instability for the case it develops, i.e. $L e<L e_{C}(R e=25, L e=2.0)$.

Figure 5.1: Exponential flame acceleration rate $\sigma$ versus the flame propagation Reynolds number $R e$ for various Markstein numbers, $M k=0 ; \pm 0.5 ; \pm 1 ; \pm 2.38$. Equations (5.9) and (5.10) are presented by the solid/dotted and dashed lines, respectively. The dotted portions denote the break of the theory. Markers show the present simulations, triangles, as well as the previous ones, [17], circles, and [24], squares.

Figure 5.2: Exponential flame acceleration rate $\sigma$ versus the flame propagation Reynolds number, $R e$, for various Lewis numbers, $L e=0.8 ; 1 ; 1.2 ; 1.6 ; 2.0$. Equations (5.9) and (5.10) are presented by the solid/dotted and dashed lines, respectively. The dotted portions denote the break of the theory. The black solid line is related to $M k=0, \mathrm{Eq}$. (5.4). Markers show the present simulations. Overall, the same colors correspond to the same Le numbers. 
Figure 5.3: Exponential flame acceleration rate $\sigma$ versus the Lewis number, $L e$, for various fixed flame propagation Reynolds numbers: $R e=5$ (a); 10 (b); 15 (c) and 20 (d). In all the plots, Eqs. (5.9) and (5.10) are presented by the blue solid/dotted and dashed lines, respectively. The dotted black lines show Eq. (5.4). The present simulations are shown by markers, with the linear trend presented by the red totted line. The factor $\Gamma$ is given by Eq. (5.13).

Figure 5.4: Exponential flame acceleration rate $\sigma$ versus the flame propagation Reynolds number, $R e$, for various Lewis numbers, $L e=0.8 ; 1 ; 1.2 ; 1.6 ; 2.0$. Equations (5.11) and (5.12) are presented by the solid/dotted and dashed lines, respectively. The dotted portions denote the break of the theory. The black solid line is related to $M k=0$, Eq. (5.4). Markers show the present simulations. Overall, the same colors correspond to the same $L e$ numbers. The factor $M k_{e f f}$ is given by Eq. (5.13), where $\Gamma=0.001$ and $n=5.5$.

Figure 5.5: Exponential flame acceleration rate $\sigma$ versus the Lewis number, $L e$, for various fixed flame propagation Reynolds numbers: $R e=5$ (a); 10 (b); 15 (c) and 20 (d). In all the plots, Eqs. (5.11) and (5.12) are presented by the blue solid/dotted and dashed lines, respectively. The dotted black lines show Eq. (5.4). The present simulations are shown by markers, with the liner trend presented by the red dotted line. The factor $M k_{e f f}$ is given by Eq. (5.13), where $\Gamma=0.001$ and $n=5.5$.

Figure 5.6: Exponential flame acceleration rate $\sigma$ versus the Reynolds number for various fixed Lewis number values of simulation data. Same configuration of model equation (5.38) fitted in order to be compared to the simulation data.

Figure 5.7: $\quad$ Term $\epsilon \sigma_{1} / \sigma_{0}$ versus Reynolds number.

Figure 6.1: Images of various flame propagation modes for equivalence ratio values $\Phi=0.8$, $0.9,1.1,1.2$, and 1.3 , as well as flow velocity values $\mathrm{u}=0.23,0.45,0.65,0.75,0.90$, and 0.95 $\mathrm{m} / \mathrm{s}[49]$.

Figure 6.2: Experimental OH PLIF measurements for propane, methane, and hydrogen mixtures. The image widths correspond to $3 \mathrm{~cm}[50]$. 


\section{List of Tables}

Table 3.1: Resolution tests for $M a=0.005, \Theta=8$. The notation for the scaled variables reads the $\Delta z_{f} / L_{f}$ being the mesh size of the grid, $U_{\max } / S_{L}$ being the maximum flame tip velocity, and $t_{\max } S_{L} / R$ - the time corresponding to the maximum flame tip velocity [46].

Table 4.1: The critical Lewis number as a stability limit of trough formation, listed for various Re values.

Table 5.1: Coupling between the Markstein, $M k$, and Lewis, Le, numbers according to Eq. (2.2) with $h(\vartheta)=1$.

Table 5.2: Term $\epsilon \sigma_{1} / \sigma_{0}$ in Eq. (5.35) for varying Re number. 


\section{Chapter 1: Introduction}

\subsection{Motivation and Literature Review on the Flame Acceleration Mechanisms}

The dynamics and morphology of accelerating flames have extensively been investigated in a multitude of configurations [1]. In this respect, pipes and slits have been one of the preferred geometries for fundamental combustion studies as it allows reasonable simplifications in the analysis. On the other hand, combustion in tubes is associated with numerous practical applications of variety of scales, such as pseudo-combustion of thermo-power waves in nanotubes [2], micro- and mesa-combustors [3], and fire safety issues in mines [4]. Various experimental studies have observed spontaneous flame acceleration, potentially followed by a deflagration-to-detonation transition (DDT) event in the geometry of flames spreading in a pipe or gap [5-14].

While such practical demands have stimulated the research in this direction for decades, until recently there was a limited theoretical understanding of the flame acceleration mechanisms. Namely, the same scenario of the conceptually laminar flame acceleration towards the detonation triggering has been demonstrated in the recent computational and analytical studies of flames in tubes/channels [15-20]. To be specific, a flame-generated flow in pipes becomes non-uniform due to the non-slip boundary conditions at the walls, hence the flame front is distorted acquiring a convex shape. This scenario is modeled by the renowned Shelkin mechanism [21,22], see Fig. 1.1. On the basis of this simple model, Bychkov et al. [17] have developed a quantitative analytical theory of flame acceleration from a closed end of a twodimensional (2D) channel with non-slip, adiabatic walls and predicted the main tendencies of the acceleration process. According to [17], at the initial, almost incompressible stage, flames in pipes accelerate exponentially until compressibility effects become of importance. The analytical formulas for the exponential acceleration rate, the flame shape and propagation speed, as well as the flame-generated flow velocity profile have been derived [17]. Akkerman et al. [19] have subsequently extended the formulation of [17] from 2D channels to axisymmetric, cylindrical tubes. The analysis [17] has also subsequently been extended to account for viscous heating [23] and gas compression [24-25], with the saturation tendencies identified when the flame speed approaches the Champan-Jouguet deflagration speed. As a result, the entire DDT scenario has 
been elucidated up to the possibility of explosion triggering in the preheated fuel mixture because of the flame acceleration $[17,19,23,26]$.

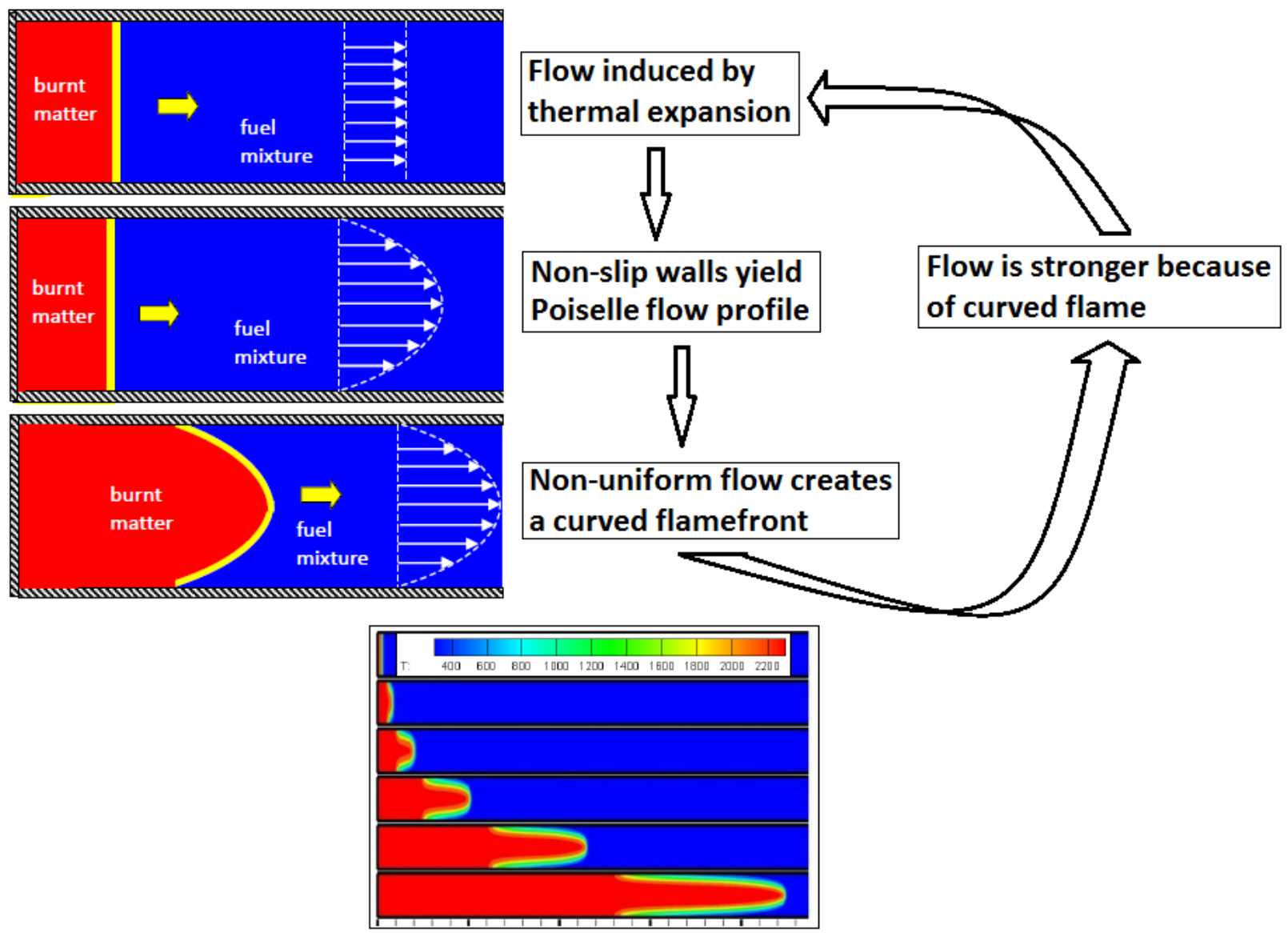

Figure 1.1: A schematic of wall-friction (a.k.a. Shelkin) mechanism, yielding an exponential acceleration regime.

While the theoretical endeavors in $[17,19]$ were substantiated by computational simulations, both of the formulations adopted a set of simplifying assumptions. One of them is the so-called Landau limit of zero flame thickness, which has been a conventional approximation used in a multitude of theoretical models. However, the impact of the transport phenomena within the burning zone, including thermal and molecular diffusivities, may appear as strong as that of viscosity, even yielding locally unstable burning regime of flame propagation. Indeed, the possibility of onset and development of multidimensional combustion instabilities, bending flame fronts, are influenced by the finite flame thickness [1,27]. In this respect, the diffusionalthermal $(D T)$ instability mode has been extensively studied in [28-32], with the (in)stability domains for non-equidiffusive flames being identified [32-35]. For a summary of computational 
studies on the DT instability, the reader is referred to [36-37], and the references therein. A conventional measure of the combustion "non-equidiffusivity" is the Lewis number $(L e)$ yielding the mass to thermal diffusivities ratio, with $L e=1$ for equidiffusive flames. Besides, the Lewis number can also be coupled to the Schmidt $(S c)$ and Prandtl $(P r)$ numbers as $L e=S c / P r$. It should be mentioned, in this light, that previous computational simulations of premixed flames in channels with non-slip adiabatic walls [17] have been performed for equidiffusive flames only, i.e. for $L e=1$.

In spite of a good quantitative agreement between the theory and simulations [17], certain specific features of accelerating flames observed numerically have not been explained by the theory. Specifically, numerical modeling has demonstrated a little trough at the flame front close to the pipe axis, whereas the theory predicted a flat flame top, see Fig. 1.2. In [17], it was pointed out that such appearance of a trough at a flame front might be related to the Darrieus-Landau $(D L)$ combustion instability that develops at the locally planar part of the flame front close to the axis, and this effect was expected to be more profound for non-equidiffusive flames, when the $D T$ instability mode comes into play too. Furthermore, it is observed that the flame isotherms get thicker close to the walls whereas theory employed infinitely thin flames (Landau limit). The $D L$ instability has been ignored in the formulation [17], due to the dominance of the Shelkin acceleration mechanism over the relatively weak instability. In contrast, the $D T$ instability mode, whose onset is triggered by non-unity Lewis numbers, $L e$, and negative Markstein numbers, $M k$, dominates on small scales, and therefore has to be considered.

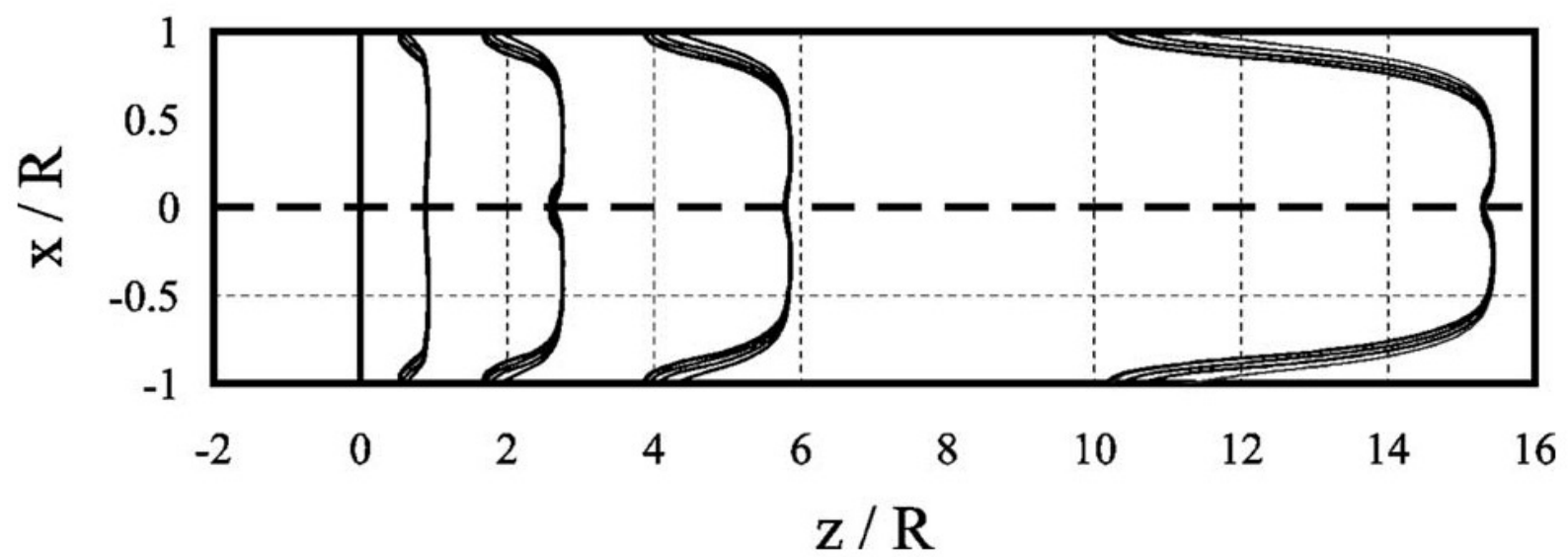

Figure 1.2: Evolution of the flame isotherms, from 600 to $2100 \mathrm{~K}$ with the step of $300 \mathrm{~K}$, in the simulation run for $\operatorname{Re}=25$, and $\operatorname{Pr}=1$ [17]. 


\subsection{Methodology}

In the present work, the theory (based on the Landau limit) and simulations (employing Le $=1$ flames) in [17] is extended further in order to incorporate the effects of internal thermaldiffusive flame phenomena on the flame acceleration scenario in channels. Firstly, realizing that the internal flame structure is crucially interrelated to diffusive properties, the coupling mechanisms in the Ze-Le- $\Theta$ parametric space is investigated in Ch. 2, for the sake of describing the stability limits for the flame front. Here, $Z e$ is the Zel'dovich number, and $\Theta$ is the density drop parameter at the flame front. Then, the critical conditions for the $D T$ flame instability is defined in terms of the critical parametric space formed by the critical Markstein $\left(M k_{C}\right)$, Zel'dovich $\left(Z e_{C}\right)$ and Lewis $\left(L e_{C}\right)$ numbers, as well as the critical activation energy $\left(E_{A, C}\right)$.

Furthermore, in contrast with the equidiffusive modeling of [17], direct numerical simulations of non-equidiffusive flames are performed, in order to explore the flame stretch and thermal-diffusive properties in a profound manner. In Ch. 3, key parameters are introduced, and the simulation mechanisms along with basic numerical equations that are used to scrutinize and quantify the flame acceleration scenario are described. Then, the simulation results are presented in Ch. 4.

In Ch. 5, two distinguished analytical models are proposed in order to complement the computational findings. Specifically, the effects of internal flame structure are incorporated via extending the theory based on the Landau limit of zero flame thickness, and the impact of diffusive properties on the non-equidiffusive flames is scrutinized. Namely, a model is developed to employ the classical flame stretch formulation of Matalon \& Matkowsky [38] into the present analysis of the flame acceleration. The thermal-diffusive and stretch effects are incorporated into the formulas for the flame acceleration rate by means of $M k$ as a thermal-chemical function, characterizing the flame response to curvature and stretch and coupled to Le [38-39]. It is recalled that $M k$ may appear positive or negative, thereby moderating or prompting the flame acceleration process, respectively [40-42]. As a review on the stretch effect in tubular flames, see, for instance, [43] and references therein. Moreover, a secondary model formulation is also developed which scrutinize the flame stretch formulations of [38], and solves the flame evolution equation via introducing the finite flame thickness effects in the form of a perturbative 
correction. As a result, the formulas for the flame acceleration rate are presented, in which the effects of the internal flame structure are again incorporated through the $M k$ number.

For both analytic approaches, another useful parameter characterizing the flame acceleration is the Reynolds number associated with the laminar flame propagation, $R e=R S_{L} / v$, which is a combination of the quantities describing the configuration (the tube radius or the channel half-width, $R$ ), the flame (the unstretched laminar flame speed, $S_{L}$ ), and the fluid (the kinematic viscosity, $v$ ). In narrow pipes, with small $R e$, where the internal flame features are apparent, the $M k$-induced modifications of the flame acceleration rate agree well with the previous simulation results, thereby justifying an undertaken improvement for the previous theory [17]. The role of the stretch diminishes with the increase in Re such that both the theory [17] and the present formulation will coincide in the limit of $R e \gg>1$. Besides, the predictions of the theory are compared to the simulation results of $\mathrm{Ch} .4$ for the non-equidiffusive flames.

Finally, in Ch. 6, the findings of computational and analytical results are discussed, and compared to each other. 


\section{Chapter 2: Zero-Dimensional Theory}

In the introductory chapter, it was mentioned that previous simulation results had minor mismatch with the theory, accompanied by morphological deformations (e.g. trough formations) of the flame front, which are presumed to be a fingerprint of the $D T$ instability. In order to define the stability limits for such a case, let us now consider a flame with $T_{f}$ and $\rho_{f}$ being the fuel temperature and density; and $T_{b}$ and $\rho_{b}$ being those for the burnt matter. Then, the thermal expansion ratio is defined as $\Theta=T_{b} / T_{f}=\rho_{f} / \rho_{b}$, and the Zel'dovich number, $Z e$, is defined in terms of the activation energy, $E_{A}$, the universal gas constant $R_{u}$, the fuel temperature, $T_{f}$, and that of the burnt matter, $T_{b}$ as [39]:

$$
Z e=\frac{\Theta-1}{\Theta} \frac{E_{A}}{R_{u} T_{b}}=\frac{\Theta-1}{\Theta^{2}} \frac{E_{A}}{R_{u} T_{f}} .
$$

The Markstein number, describing the flame response to curvature and stretch, is coupled to other thermal-chemical flame properties $(Z e, L e$, and $\Theta)$ as [39]:

$$
M k=\frac{\Theta}{\Theta-1} \int_{1}^{\Theta} \frac{h(\vartheta)}{\vartheta} d \vartheta-\frac{1}{2} \frac{Z e(1-L e)}{\Theta-1} \int_{1}^{\Theta} \frac{h(\vartheta)}{\vartheta} \ln \left(\frac{\Theta-1}{\vartheta-1}\right) d \vartheta,
$$

where, $\vartheta=T / T_{f}$ is the scaled temperature such that $1 \leq \vartheta \leq \Theta$, and $h(\vartheta)$ is the function describing the temperature-dependence of the transport coefficients. Then, with the following designations

$$
\begin{gathered}
J_{1}=\int_{1}^{\Theta} \frac{h(\vartheta)}{\vartheta} d \vartheta \\
J_{2}=\int_{1}^{\Theta} \frac{h(\vartheta)}{\vartheta} \ln \left(\frac{\Theta-1}{\vartheta-1}\right) d \vartheta
\end{gathered}
$$

Eq. (2.2) can also be expressed as

$$
L e=1-\frac{2 \Theta J_{1}-2(\Theta-1) M k}{Z e J_{2}} .
$$

\subsection{Critical Parameters of the Diffusional-Thermal (DT) Instability}

Recalling that the $D T$ instability develops for $M k<0$, one can define the critical value of the Lewis number, $L e_{C}$, associated with $M k=0$, and thereby being threshold for the $D T$ instability. Specifically, the $D T$ instability develops if $L e<L e_{C}$, with 


$$
L e_{C}=L e(M k=0)=1-\frac{2 \Theta J_{1}}{Z e J_{2}} .
$$

Now it is also the time to recall the physical constraint for the lower limit of the Lewis number; namely, $L e>0$ in the reality. Hence, the situation when the threshold Le formally appears nonpositive, $L e_{C} \leq 0$, simply denotes that the flame is absolutely stable against the $D T$ instability in that case. Thus, the critical Zel'dovich number associated with such a limit of absolute stability can be defined. The quantity will obviously be related to both zero $L e$ and $M k$, namely, Eq. (2.6) yields:

$$
Z e_{C}=Z e(L e=0, M k=0)=Z e\left(L e_{C}=0\right)=2 \Theta J_{1} / J_{2} .
$$

Then, with Eq. (2.7), Eq. (2.6) takes the form

$$
L e_{C}=1-\frac{Z e_{C}}{Z e} .
$$

One should recall, again, that for $Z e \leq Z e_{C}$ one has $L e_{C} \leq 0$, which is impossible. In other words, for $Z e \leq Z e_{C}$, the flame is unstable only for $L e<L e_{C}$, which is a negative number anyway. Therefore, it can be concluded that the flame front never becomes unstable in this case, and the condition that a flame is absolutely stable against the DT instability can be stated as:

$$
Z e \leq Z e_{C}=2 \Theta J_{1} / J_{2}
$$

or

$$
E_{A} \leq E_{A, C}=\frac{2 \Theta^{3} R_{u} T_{f}}{\Theta-1} \frac{J_{1}}{J_{2}} .
$$

\subsection{Neutral Curves}

As a result, it is identified that the $D T$ instability may develop only if $Z e>Z e_{C}, E_{A}>E_{A, C}$, and $L e<L e_{C}=1-Z e_{C} / Z e$. The associated neutral curves are shown in Figs. 2.1 and 2.2, which are the outcomes of Eqs. (2.8) and (2.9). In this regard, two distinct functional temperaturedependences of the transport coefficients are chosen for convenience, namely $h(\vartheta)=1$, and $h(\vartheta)$ $=\vartheta^{1 / 2}$. The integral $J_{2}$ has been taken numerically via conventional trapezoidal integration.

It is evident from Fig. 2.1 that increasing $\Theta$ extends the stability regime for the flame, which can also be verified in Fig. 2.2 as the instability domain reduces with increasing $\Theta$. Similarly, Fig. 2.3 shows that $L e_{C}$, needed to prevent the instability, gets larger as $Z e$ grows. 


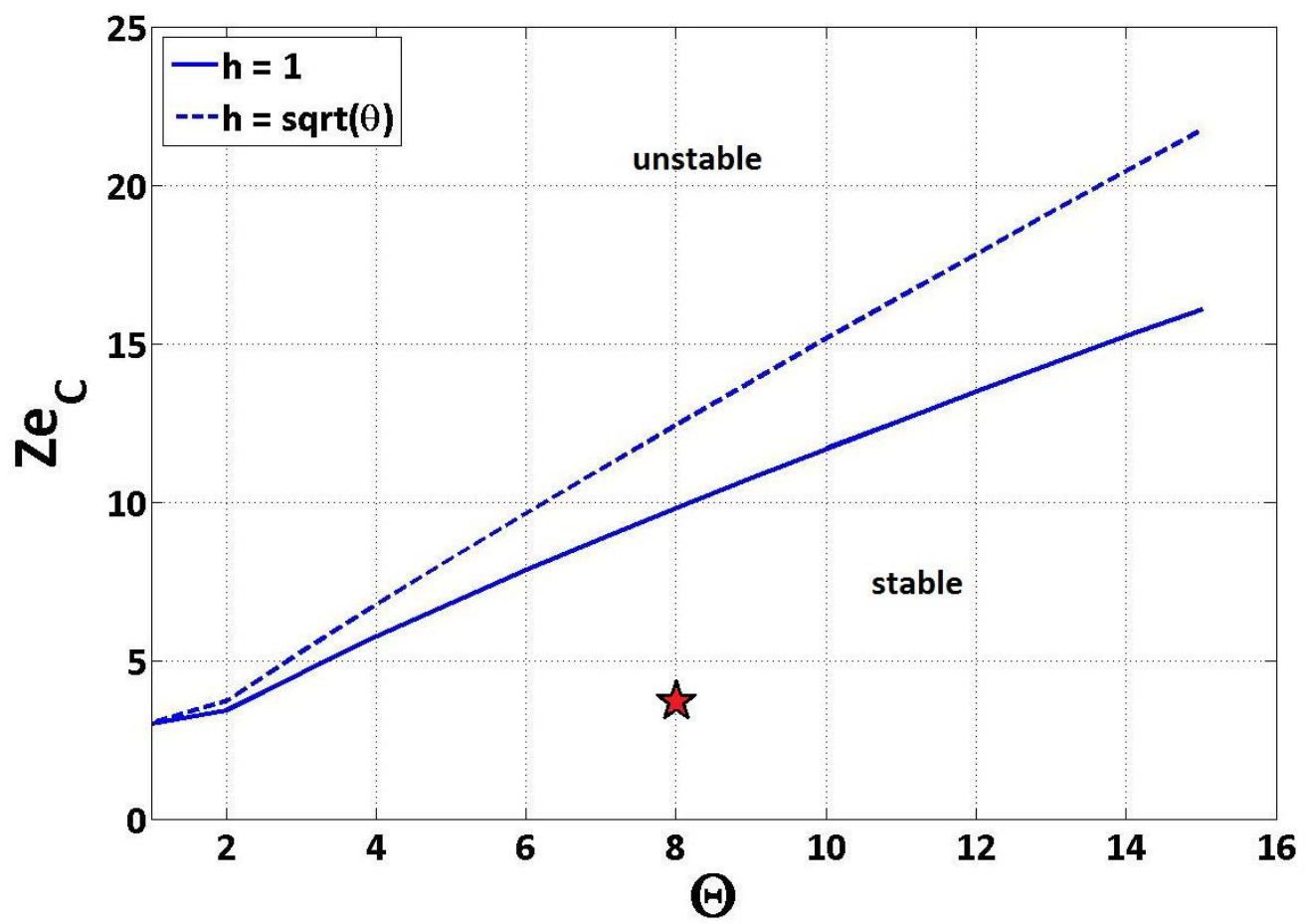

Figure 2.1: $Z e_{C}$ as a function of $\Theta$ for $h(\vartheta)=1$ (dashed), and $h(\vartheta)=\vartheta^{1 / 2}$ (solid). Marker correspond to current simulation parameters.

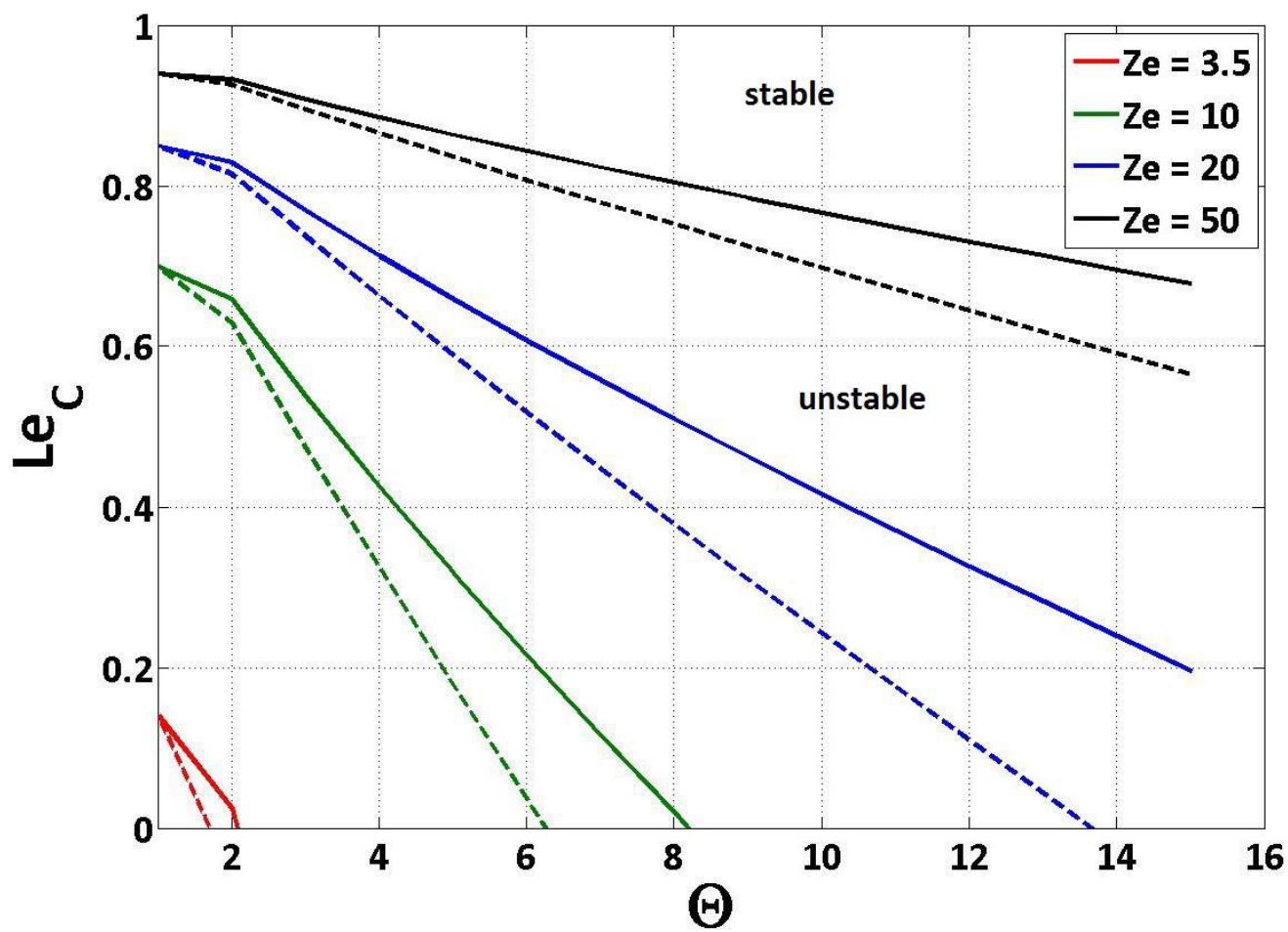

Figure 2.2: $L e_{C}$ versus $\Theta$ for fixed $Z e=[3.5,10,20,50]$. Solid lines use $h(\vartheta)=1$, and dashed lines use $h(\vartheta)=\vartheta^{1 / 2}$. 


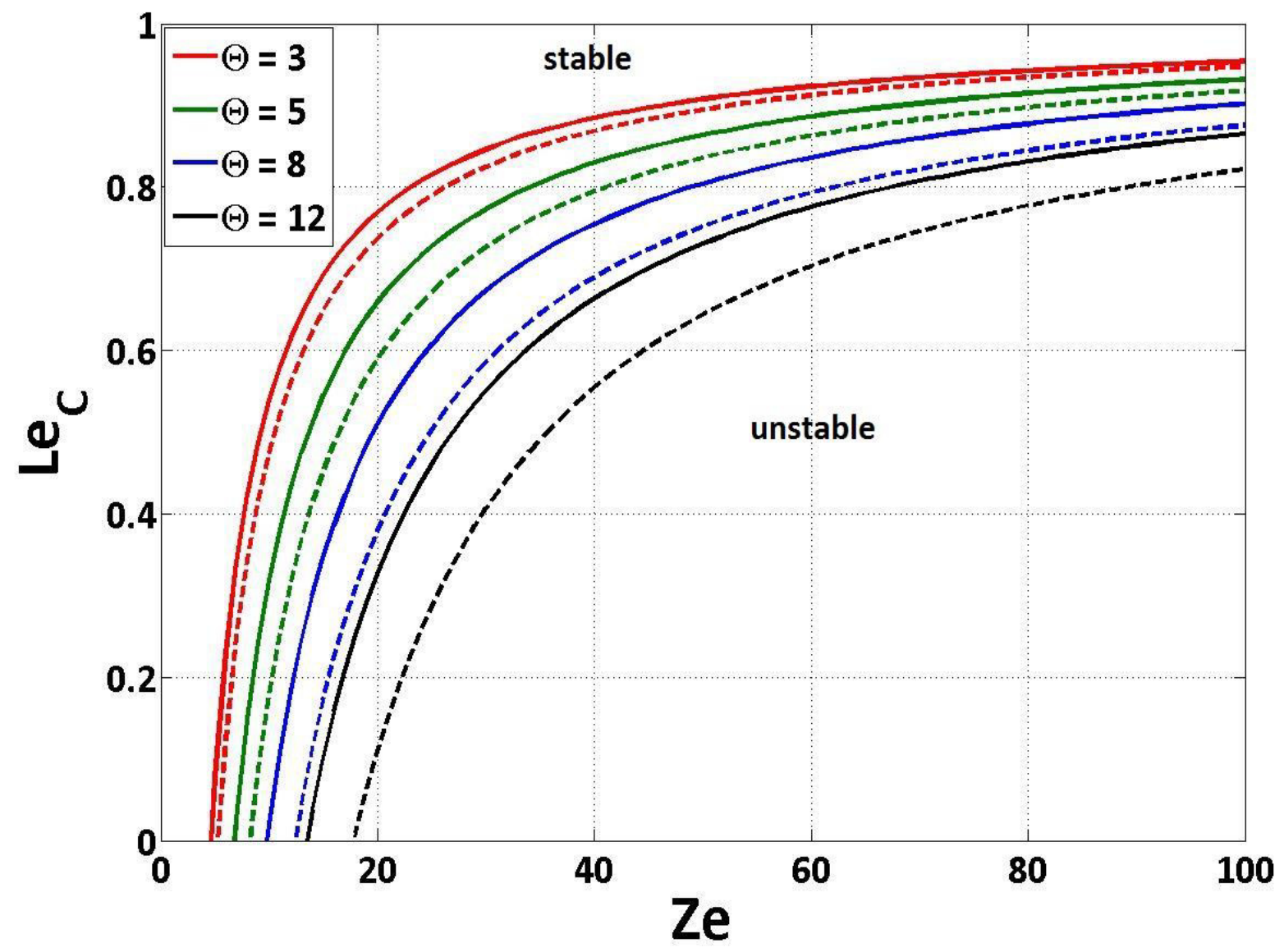

Figure 2.3: $L e_{C}$ versus $Z e$ for fixed $\Theta=[3,5,8,12]$. Solid lines use $h(\vartheta)=1$, and dashed lines use $h(\vartheta)=\vartheta^{1 / 2}$.

Overall, the analysis of this chapter yields the absolute stability limits for a flame against the $D T$ instability. In the following chapters, numerical simulations will be performed where the Zel'dovich number is set to $Z e=3.5$, which is lower than $Z e_{C}$ corresponding to the given thermal expansion rate, which is picked as $\Theta=8$ in simulations (see the red marker in Fig. 2.1). Hence, the critical Lewis number for the simulation runs corresponds to a negative number. As previously explained, in this respect, the $D T$ instability is not expected to develop, however the disturbances on flame front are expected to be observed, which may still be attributed as an instability finger-print. 


\section{Chapter 3: Description of the Numerical Approach}

In this chapter, the description of the fully-compressible, finite-volume direct numerical simulations of the Navier-Stokes equations "in-house" solver is given, which has been employed in the present studies on non-equidiffusive flames. The original code is developed by Dr. LarsErik Eriksson at Volvo Aero Co. (Goteborg, Sweden), and it has been subsequently revised and updated by several research groups henceforward, including Dr. M. Liberman (Uppsala University), Dr. V. Bychkov and Dr. D. Valiev (Umea University), and finally Dr. V. Akkerman (West Virginia University). Thanks to these updates, the code is able to solve the complete set of the hydrodynamic combustion equations including transport processes and chemical kinetics.

\subsection{Basic Equations}

The basic equations of continuity, momentum conservation, energy conservation, and species sonservation read

$$
\begin{gathered}
\frac{\partial \rho}{\partial t}+\frac{\partial}{\partial x_{i}}\left(\rho u_{i}\right)=0, \\
\frac{\partial}{\partial t}\left(\rho u_{i}\right)+\frac{\partial}{\partial x_{j}}\left(\rho u_{i} u_{j}+p \delta_{i j}-\gamma_{i j}\right)=0, \\
\frac{\partial}{\partial t}\left(\rho \varepsilon+\frac{1}{2} \rho u_{i} u_{i}\right)+\frac{\partial}{\partial x_{j}}\left(\rho u_{j} H+\frac{1}{2} \rho u_{i} u_{i} u_{j}+q_{j}-u_{i} \gamma_{i j}\right)=0, \\
\frac{\partial}{\partial t}(\rho Y)+\frac{\partial}{\partial x_{i}}\left(\rho u_{i} Y-\frac{\mu}{S c} \frac{\partial Y}{\partial x_{i}}\right)=-\frac{\rho Y}{\tau_{R}} \exp \left(-E_{A} / R_{u} T\right),
\end{gathered}
$$

respectively, where $Y$ is the mass fraction of the fuel mixture, $\varepsilon=Q Y+C_{V} T$ is the internal energy, $H=Q Y+C_{P} T$ is the enthalpy, $Q$ is the energy release in the reaction, $C_{V}$ and $C_{P}$ are heat capacities at constant volume and pressure, respectively. Equation (3.4) employs a one-step irreversible Arrhenius reaction of the first order, with the activation energy $E_{A}$ and the time dimension constant $\tau_{R}$. Finally, the stress tensor $\gamma_{i j}$ and the energy diffusion vector $q_{j}$ are given by

$$
\begin{gathered}
\gamma_{i j}=\zeta\left(\frac{\partial u_{i}}{\partial x_{j}}+\frac{\partial u_{j}}{\partial x_{i}}-\frac{2}{3} \frac{\partial u_{k}}{\partial x_{k}} \delta_{i j}\right), \\
q_{j}=-\zeta\left(\frac{C_{P}}{P r} \frac{\partial T}{\partial x_{j}}+\frac{Q}{S c} \frac{\partial Y}{\partial x_{j}}\right),
\end{gathered}
$$

where $\zeta$ is the dynamic viscosity. The combustible premixture conventionally consists of a diatomic perfect gas of a constant molecular weight $m=2.9 \times 10^{-2} \mathrm{~kg} / \mathrm{mol}$, with $C_{V}=5 R_{u} / 2 \mathrm{~m}, C_{P}$ 
$=7 R_{u} / 2 \mathrm{~m}$, where $R_{u}=8.314 \mathrm{~J} /(\mathrm{mol} . \mathrm{K})$ is the universal gas constant, and the equation of state is $P=\rho R_{u} T / m$. Within the current configuration, it is considered that a flame propagates in a $2 \mathrm{D}$ semi-open channel of half-width $R$, with adiabatic and no-slip boundary condition at the walls, i.e. $\boldsymbol{u}=0$ and $\widehat{\boldsymbol{n}} \cdot \boldsymbol{\nabla} T=0$, where $\widehat{\boldsymbol{n}}$ is the normal vector of wall surface. An initialy planar flame front is initiated near the closed end of the channel, and it propagates towards the open end. The initial pressure and temperature of the fuel mixture are taken to be $P_{f}=10^{5} \mathrm{~Pa}$ and $\mathrm{T}=300 \mathrm{~K}$, respectively. The thermal and chemical parameters are chosen to reproduce the most important properties of typical methane and propane laboratory flames. Namely, the dynamic viscosity is $\zeta$ $=1.7 \times 10^{-5} \mathrm{~N} . \mathrm{s} / \mathrm{m}^{2}$, and the activation energy is $E_{A}=32 R_{u} T_{f}$. Also, the Prandtl number is kept fixed at $P r=1$, such that $L e=S c$, hence is varied through $S c$. In order to avoid the influence of gas compression effects on the burning process, realistically low Mach number value was taken, namely $M a=10^{-3}$, which corresponds to the planar flame velocity of $U_{f}=34.7 \mathrm{~cm} / \mathrm{s}$. Though, it resulted in numerical difficulties since the large difference between the flame velocity and the sound speed dramatically increases the computation time. Finally, the flame thickness used in the calculations is conventionally defined as

$$
L_{f}=\frac{v}{\operatorname{Pr} U_{f}} .
$$

The thermal expansion in the burning process is determined by the energy release in the reaction, and its initial quantity in all the simulations was kept as large as $\Theta=8$ in order to obtain similar conditions as typical methane and propane burning.

\subsection{Numerical Scheme}

The numerical scheme of this finite-volume Navier-Stokes solver is second-order accurate in time, fourth-order accurate in space for the convective terms, and second-order accurate in space for the diffusive term. The computational methods are thoroughly described, in particular in [44-45], and it has been utilized successfully in the studies of laminar burning, hydrodynamic flame instabilities, development of corrugated flames, and other related phenomena [17-19, 4445]. In the present simulations, the channel width $(2 R)$ is varied within the range $20 L_{f}-70 L_{f}$, and the aspect ratio is taken of the order of $10^{3}$ such that the channel length practically does not influence the results. 


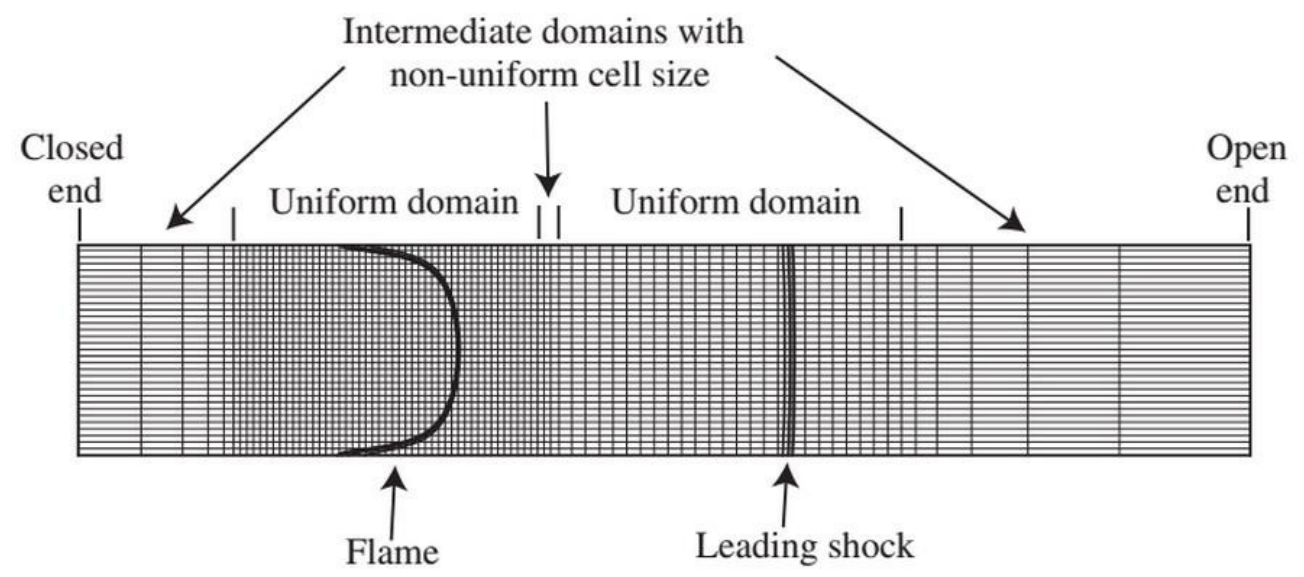

Figure 3.1: A sketch of the adaptive non-uniform grid with variable resolution [46].

The mesh is composed of a structured rectangular grid, which is adaptive to the flame propagation. In order to reduce the computation time to reasonable intervals, the grid is made non-uniform in the (axial) $z$-direction, where the fine resolution region lies around the flame front. A scheme of a typical grid used in the solver can be seen in Fig. 3.1 [46]. In this zone, the grid size is $0.2 L_{f}$ in the $z$-direction, which is good enough to resolve the internal flame structure. Outside this fine grid region the mesh size grows gradually with circa $2 \%$ change in size between adjacent cells. In order to keep the flame in the fine grid zone, adaptive mesh is applied which moves together with the flame. On the other hand, the grid along the $x$-axis is taken to be uniform, with the cell size comparable to $0.5 L_{f}$. Hence, the zone of large velocity gradients close to the wall were able to be resolved quite well.

\begin{tabular}{lllllll}
\hline$\Delta z_{f} / L_{f}$ & $U_{\max } / S_{L}$ & $\Delta U_{\max } / S_{L}$ & $t_{\max } S_{L} / R$ & $\Delta t_{\max } S_{L} / R$ & $Z_{\text {tip } * *} / R$ & $\Delta Z_{\text {tip }, *} / R$ \\
\hline 1.0 & 31.14 & & 0.3265 & & 4.5095 & \\
0.5 & 34.68 & 3.54 & 0.3032 & 0.2706 & 5.282 & 0.7725 \\
0.25 & 36.171 & 1.43 & 0.2973 & 0.0059 & 5.441 & 0.159 \\
0.125 & 36.85 & 0.679 & 0.2965 & 0.0008 & 5.474 & 0.033 \\
\hline
\end{tabular}

Table 3.1: Resolution tests for $M a=0.005, \Theta=8$. The notation for the scaled variables reads the $\Delta z_{f} / L_{f}$ being the mesh size of the grid, $U_{\max } / S_{L}$ being the maximum flame tip velocity, and $t_{\max } S_{L} / R$ being the time corresponding to the maximum flame tip velocity [46]. 


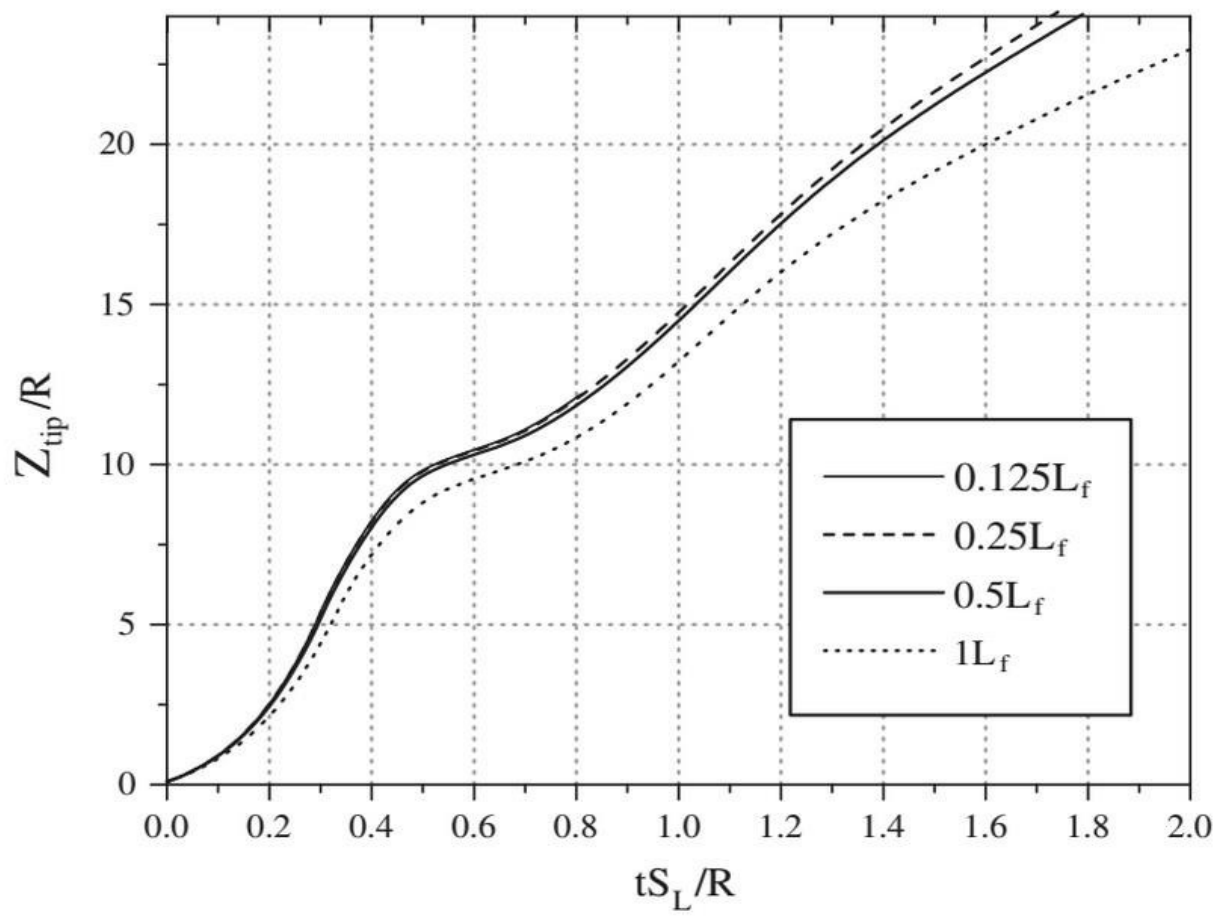

Figure 3.2: A resolution test for the flame tip position versus time, where different mesh sizes are considered which are scaled with respect to the flame thickness [46]. $L e=1$.



Figure 3.3: A resolution test for the flame tip position versus time, similar to Fig. (3.2), but for the current simulations. $L e=0.2$. 
In order to check whether the number of cells were sufficient for the problem, various test simulations were run with the number of cells increased 3 times in $x$-direction which yielded an accuracy of (5-10)\%, and this can be taken as the numerical accuracy of the simulation data. Such a test conducted in previous studies can be seen in Table 3.1 and Fig. 3.2 [46], and the test conducted for the current solver can be seen in Fig. 3.3. Similar to [44-46], in the present work, the Zel'dovich-Frank-Kamenetsky solution for a planar flame front has been employed as an initial condition, with the planar flame front initiated at a distance $4 L_{f}$ from the closed end of the channel. In the reference frame moving with the flame front, the initial conditions read:

$$
\begin{array}{r}
\frac{T(z)}{T_{f}}= \begin{cases}1+(\Theta-1) \exp \left(-z / L_{f}\right), & z \geq 0 \\
\Theta, & \mathrm{z}<0\end{cases} \\
\frac{P(z)-P_{f}}{\rho_{f} S_{L}{ }^{2}}= \begin{cases}(4 / 3 \operatorname{Pr}-1)(\Theta-1) \exp \left(-z / L_{f}\right), & z \geq 0 \\
(\Theta-1), & \mathrm{z}<0\end{cases}
\end{array}
$$

with

$$
\frac{u_{z}(z)}{S_{L}}=\frac{\rho_{f}}{\rho(z)}=\frac{T}{T_{f}}, \quad \text { and } \quad Y(z)=\frac{\Theta-T / T_{f}}{\Theta-1} .
$$

Likewise, the non-reflecting boundary conditions in [44-46] are adopted at the open end of the channel in order to avoid reflections of weak shocks and sound waves that may influence the burning and acceleration process.

The dimensionless parameter of the problem that describes the flame dynamics is the flame propagation Reynolds number defined as $R e=R U_{f} / v$, which can be coupled to the flame thickness through scaled tube half-width as follows:

$$
R e=\frac{R U_{f}}{v}=\frac{R}{\operatorname{Pr} L_{f}} .
$$

Since $\operatorname{Pr}=1, \operatorname{Re}$ is varied directly through the channel half-width within the limits $R e=5 \sim 35$. The flow ahead of the flame, however, is described by another Reynolds combination:

$$
R e_{\text {flow }}=\frac{2 R\left\langle u_{z}\right\rangle}{v}=2(\Theta-1) \operatorname{Re} \frac{U_{w}}{U_{f}},
$$

where the average flow velocity along $z$-direction is defined as $\left\langle u_{z}\right\rangle=(\Theta-1) U_{w}$. Unlike $R e$ of Eq. (3.11), $R e_{\text {flow }}$ of Eq. (3.12) increases as the flame accelerates. Yet, present simulations are performed where the flow Reynolds number remained within the limits of the laminar flow, i.e. $R e_{\text {flow }}<10^{3}$. Hence, transition to turbulent flow is beyond the scope of the present work. 


\section{Chapter 4: Simulation Results}

In this chapter, the effects of diffusive properties on the flame morphology and dynamics are scrutinized and quantified in a detailed parametric study, where diffusive properties are represented by $L e$. In order to be realistic, $L e$ is varied within the range of $0.2-2.0$, with $L e<1$ implying that mass diffusion dominates over the heat diffusion, and $L e>1$ meaning the opposite case. Having fixed $\operatorname{Pr}=1$, various scenarios for different $S c$ numbers are thereby investigated. Another important parameter is the flame propagation Reynolds number coupled to the flame thickness, Eq. (3.11), which is varied by the channel width throughout the simulations.

\subsection{Equidiffusive Flames}

A typical evolution of an equidiffusive flame can be seen in Figs. 4.1 and 4.2, where the effect of the channel size on the flame morphology is evident through the formation of a little trough close to the tube axis when channel width is increased. In contrast, previous theories predicted a flat top of the flame front, with such trough formation being guessed to be either an artifact due to initial conditions, or a footprint of a combustion instability developing at the locally planar part of the flame front close to channel axis [17]. Such a deformation of the flame morphology is found to be an important effect: it alters the dynamical behavior of the flame, which is expected since increased surface area changes the burning rate. As a result, in Fig. 4.3 for the scaled flame tip velocity, the flame front surface area and the total burning rate, a deviation from the exponential burning regime is observed. Besides, due to the flame surface deformation, one can also see a discrepancy between these three curves despite they are predicted to coincide via the same exponent rate by the previous theory. Here, one should recall that the burning rate is traditionally measured through an increase in the flame surface area (or length, in a 2D case). However, this method yields inaccurate results for strongly corrugated flame fronts with troughs and crests, since the local normal velocity significantly differs from the planar flame velocity. Therefore, the burning rate here is calculated as $[47,48]$

$$
U_{w}=\frac{1}{\rho_{f} R} \int \frac{\rho Y}{\tau_{R}} \exp \left(-E_{A} / R_{u} T\right) d x d z
$$



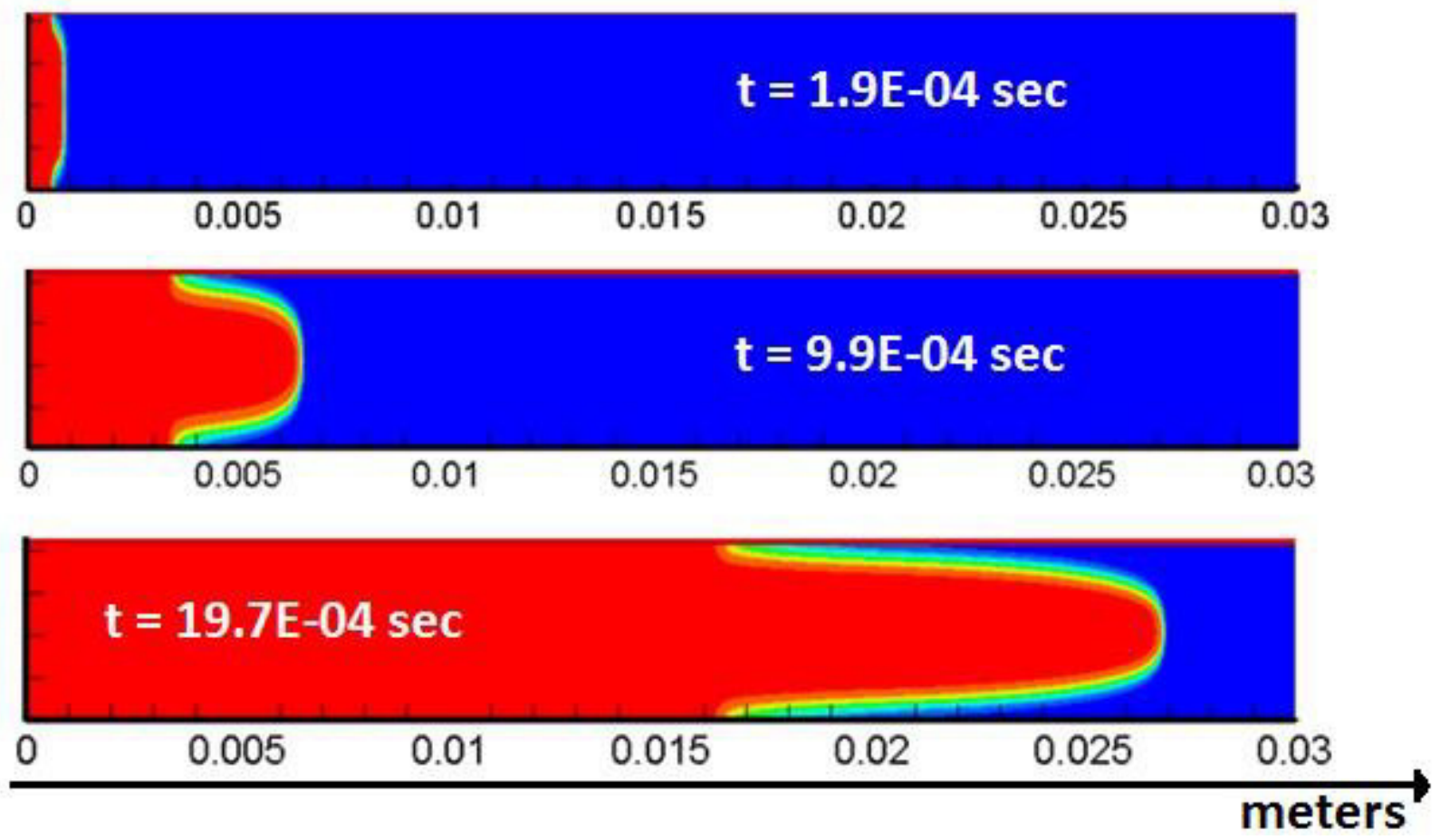

Figure 4.1: Color temperature snapshots for flame propagation in channels with $L e=1, R e=10$.
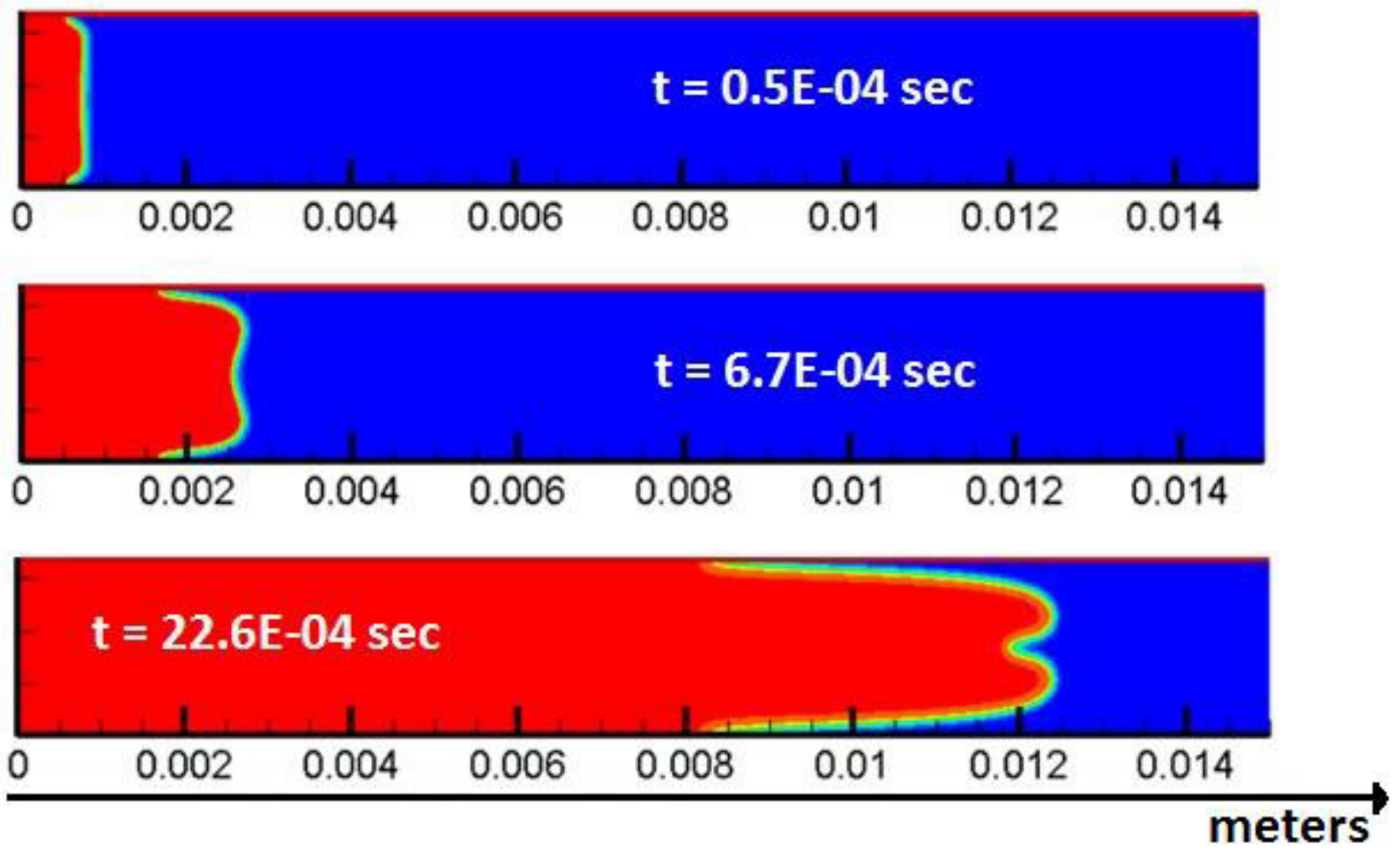

Figure 4.2: Color temperature snapshots for flame propagation in channels with $L e=1, R e=20$. 

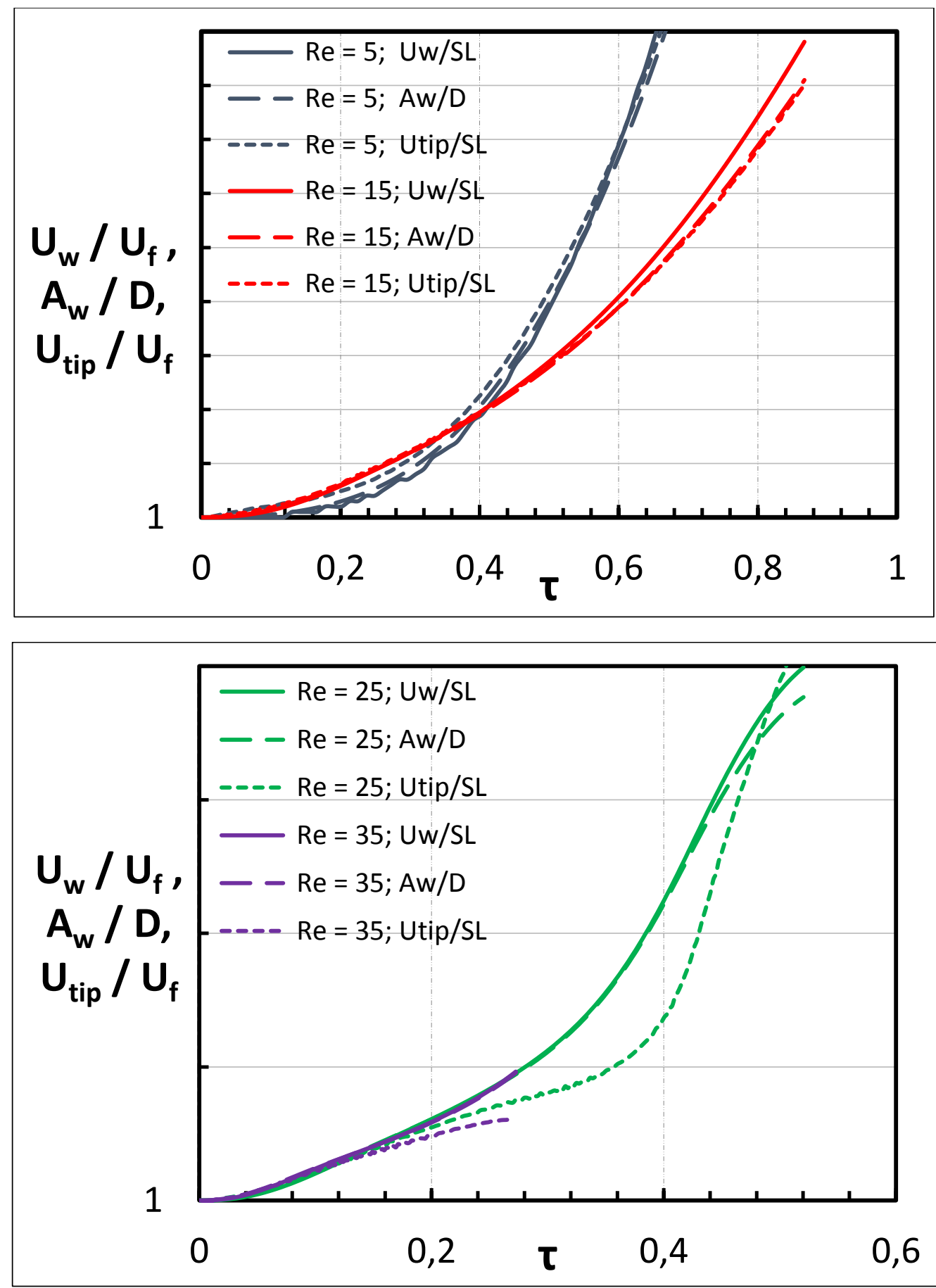

Figure 4.3: The scaled flame tip velocity, $U_{t i p} / U_{f}$, the surface area, $A_{w} / D$, and the scaled total burning rate, $U_{w} / U_{f}$, versus the scaled time for $L e=1$ and $R e=5-35$.

The non-exponential behavior due to the increased channel width is more visible in the semi-logarithmic scale, Fig 4.4, where the exponential regression lines are added to measure the 
exponential regime acceleration rate. The wider the channel, the stronger the morphological deformations cause deviation from the exponential regime of acceleration.



Figure 4.4: The scaled burning rate vs the scaled time for $L e=1$ on semi-log plot, along with exponential regression fits.

To validate the accuracy of the simulation data, the results for equidiffusive flames is compared with previous simulation results and theory [17]. In Fig. 4.5, the acceleration rate for various channel width cases are shown, where a reasonable match between different previous simulation results $[17,24]$ and present ones could be observed. 


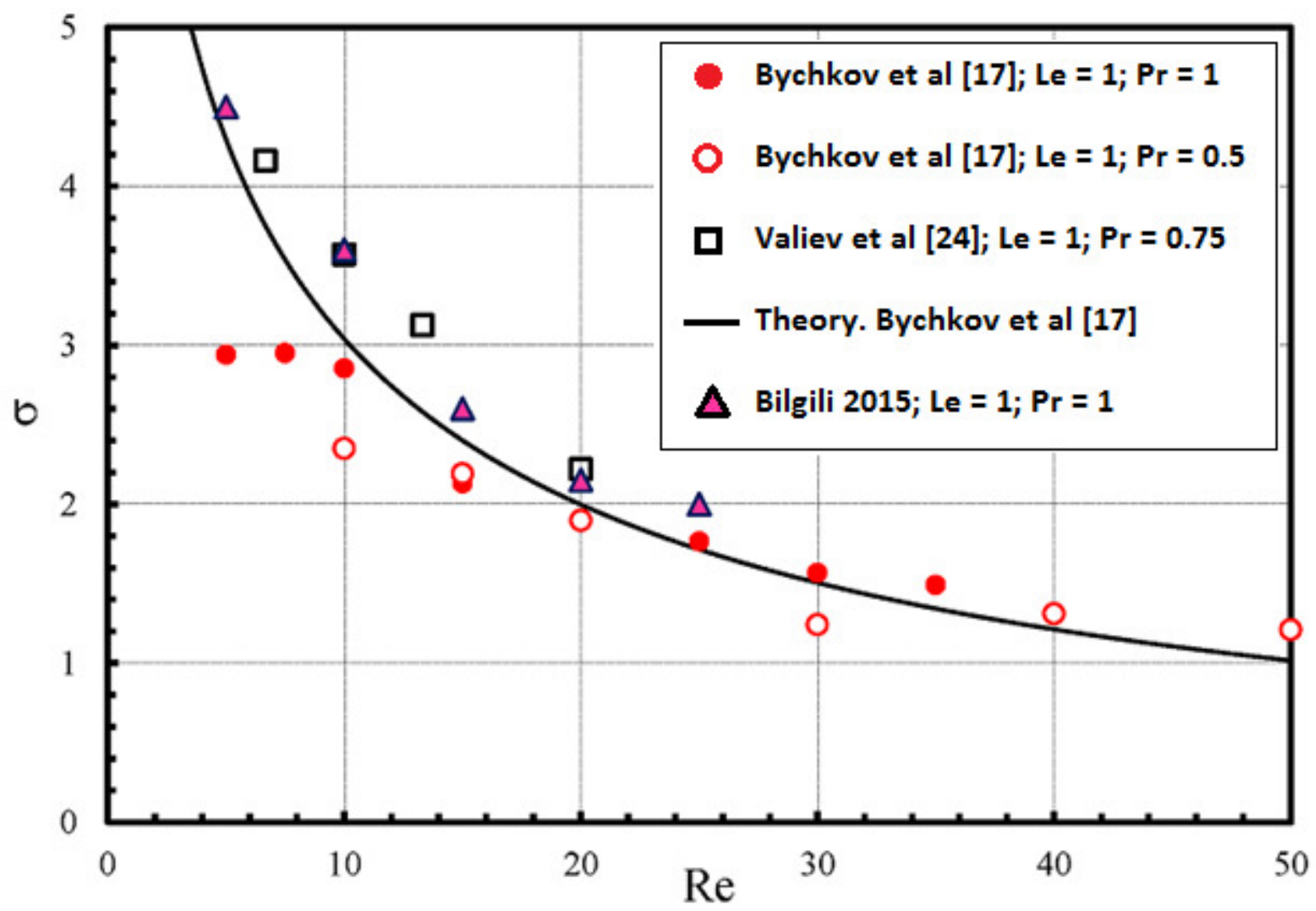

Figure 4.5: The scaled exponential flame acceleration rate versus the scaled time for $L e=1$.

\subsection{Non-Equidiffusive Flames}

To address the possible effects of diffusion properties on the flame front deformations, the scenario where the $D T$ instability modes might be entangled with the overall flame structure is put forth. Hence, the dynamics of non-equidiffusive flames is scrutinized in order to understand the role of $L e$ in the flame acceleration.

\subsubsection{Le $>1$ results}

Figures 4.6 and 4.7 show a typical evolution of a strongly non-equidiffusive flame. In contrast with Figs. 4.1 and 4.2, the trough formation is not observed even though the channel size is increased. This is a good example which reveals the nature of the Le number effects on the internal flame structure. Namely, increasing $L e$ also increases the flame thickness effectively, which in turn reduces the elasticity of the flame. Hence, if $L e$ is large enough, then the flame front becomes less prone to be corrugated and the shape of surface remains nearly convex throughout the acceleration. Moreover, increasing $L e$ effectively increases heat diffusion; hence one can observe the heated localized regions at the walls, whereas the flame front is cooler. 

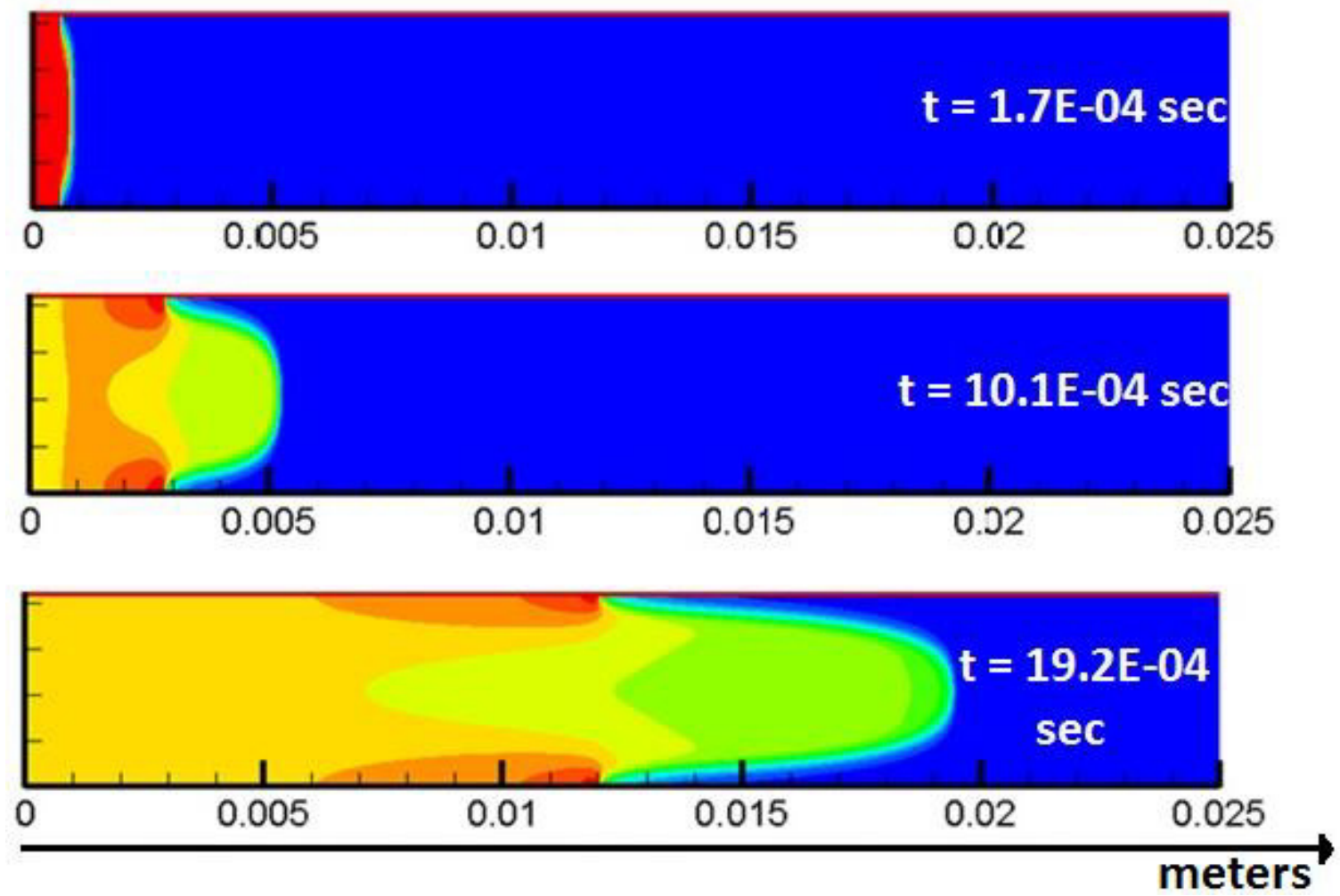

Figure 4.6: Color temperature snapshots for flame propagation in channels with $L e=2, R e=10$.


Figure 4.7: Color temperature snapshots for flame propagation in channels with $L e=2, R e=20$. 


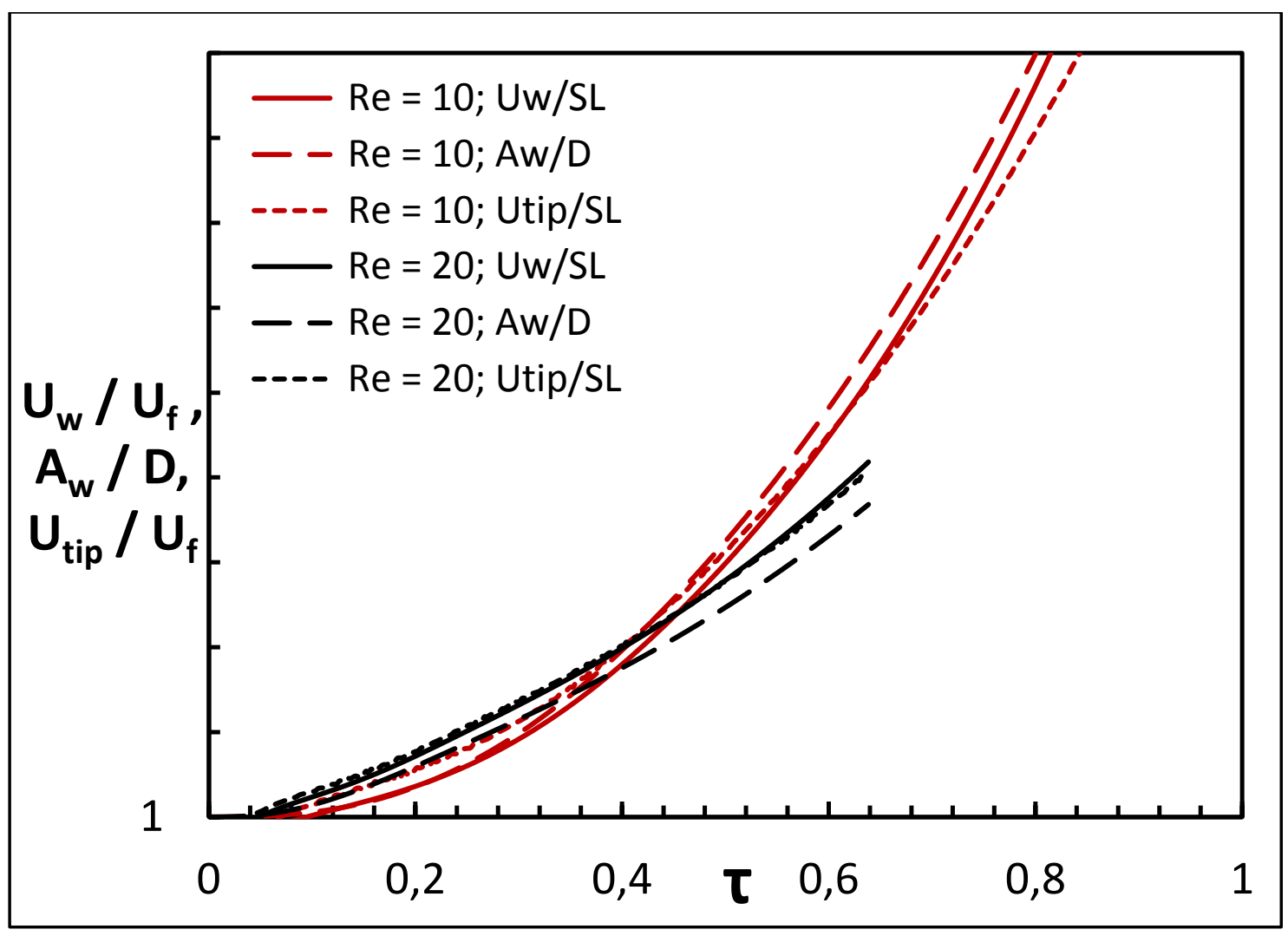

Figure 4.8: The scaled flame tip velocity, $U_{\text {tip }} / U_{f}$, the surface area, $A_{w} / D$, and the scaled total burning rate, $U_{w} / U_{f}$, versus the scaled time for $\mathrm{Le}=2.0$ and $R e=10,20$.

Nonetheless, increasing the channel size poses a similar effect by perturbing the exponential regime, which can be seen in Fig. 4.8 for the flame tip velocity, the surface area, and the total burning rate. Similarly, the deviation from exponential behavior is observed more clearly in Fig. 4.9, with the effect promoting with the increase in the channel width. Though, such deviation effects are weaker for larger Le numbers.

Overall, imposing Le effects significantly alters the flame acceleration scenario for nonequidiffusive flames. Fig. 4.10 shows the acceleration rates for various Le cases, where current simulation data are compared to the previous simulation data and theory. It is apparent that, the acceleration rate is reduced for small $R e$ values as $L e$ gets larger. This result is associated with the increased flame thickness which in turn reduces the acceleration in narrower channels. Hence, one can observe the appearance of a maximum acceleration rate for $L e>1$ cases. The bending-down behavior of the simulation data in Fig. 4.10 for small $R e$ will be further studied in Ch. 5 with respect to an analytical point of view. 


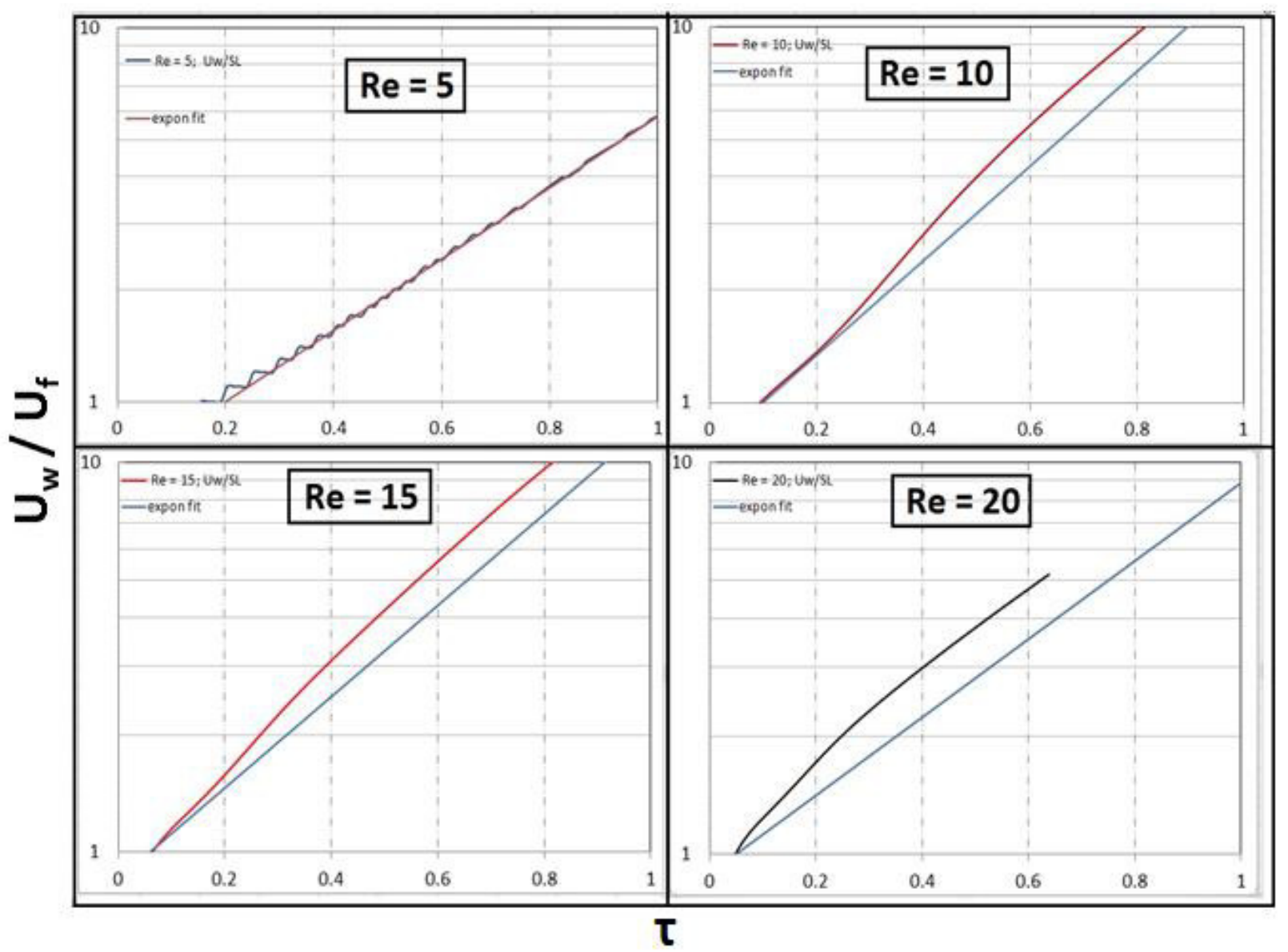

Figure 4.9: The scaled burning rate versus the scaled time for $L e=2$, along with exponential regression fits.



Figure 4.10: The scaled exponential flame acceleration rate versus the scaled time for $L e=0.8 ; 1.0 ; 1.2 ; 1.6 ; 2.0$. 


\subsubsection{Le $<1$ results}

So far, simulation data for non-equidiffusive flames had shown that the flame morphology is significantly altered by the Lewis number effects. Recalling Figs. 4.1 and 4.2, in addition to the channel width, such a trough formation might also be related to diffusive flame properties by means of heat and mass diffusion. Therefore, in the present sub-section, via gradually decreasing $L e$ in the present simulations, it is shown that such a trough formation becomes more and more concave as $L e$ gets smaller, hence increasing the surface area of the flame front, thereby causing an increase in total burning rate and promoting the acceleration.

Decreasing $L e$ further eventually escalates the trough formation and creates a split between the lower and upper crests of the flame front. Such a behavior creates a time period where the acceleration rate is substantially increased due to the positive correlation between the large increase in the flame surface area and the burning rate. Figures 4.11 and 4.12 present the evolution of the central trough for strongly non-equidiffusive flames, $L e=0.2$, for $R e=25$ (Fig. 4.11) and $R e=20$ (Fig. 4.12). It is noted that the central trough in Fig. 4.12 provides a dramatic increase in the flame surface area. Furthermore, the upper and lower crests are stretched further, and the middle trough becomes a deep well and retarded with respect to the crests. After this instant, the middle trough begins collapsing onto itself and closing the gap with a high acceleration rate at the channel axis. Such a behavior rapidly decreases the overall surface area and causes a prompt decrease in total burning rate, yielding a "slowdown" period. Yet, the momentum of the burnt matter along the channel axis that gained a substantial acceleration during the collapse of the trough nonetheless pushes the flame front, which creates a third crest along the axis, and is visible in latter snapshots in Fig. 4.11. Apparently, the formation of the third finger is possible only when the channel width is big enough, so the situation is somehow different in Fig. 4.12, where the channel is smaller. Here, for $\operatorname{Re}=20$, the third finger formation is initialized, however quickly consumed by the crests. 


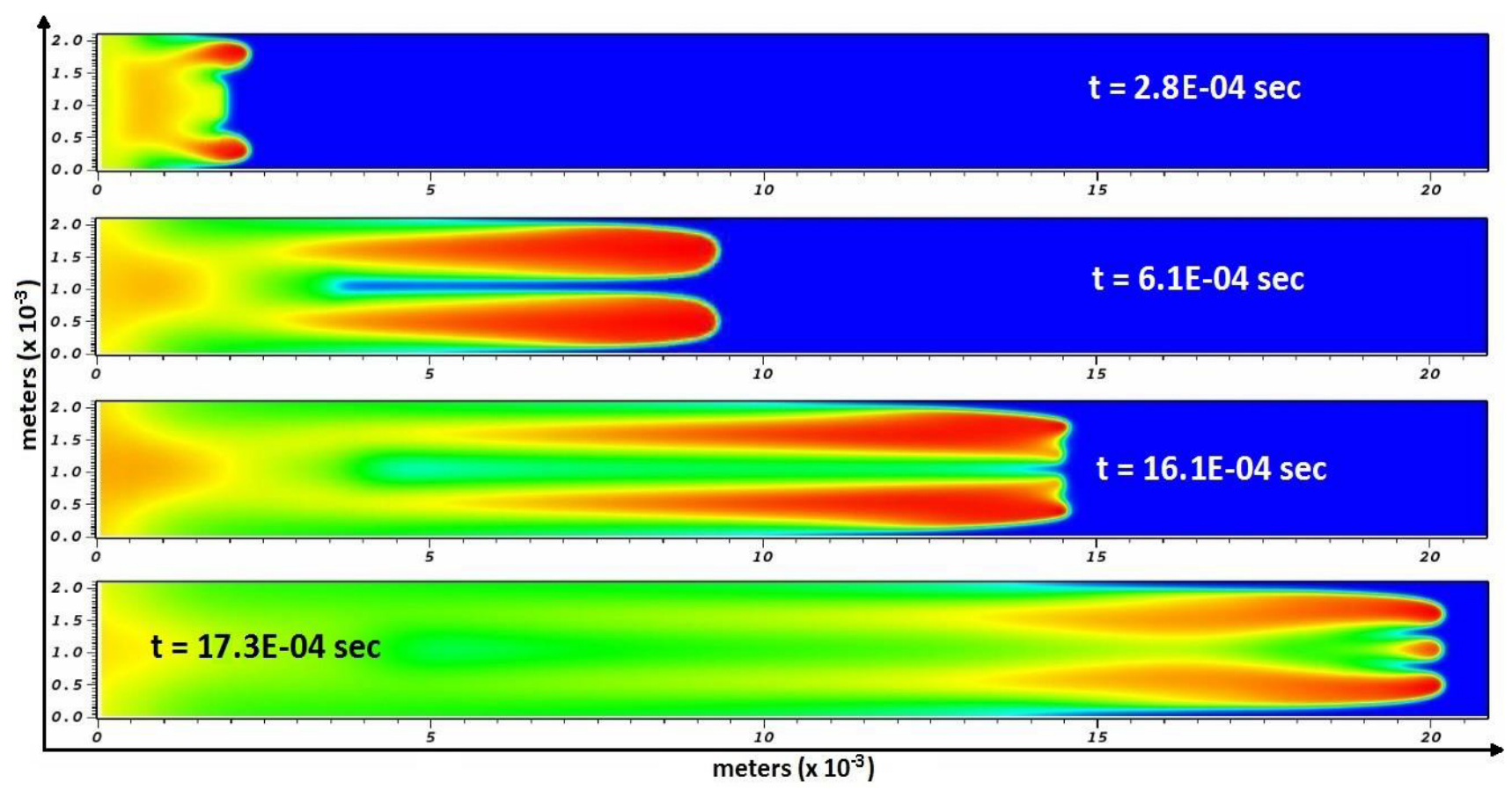

Figure 4.11: Evolution of the central trough for non-equidiffusive flames $(R e=25, L e=0.2)$.

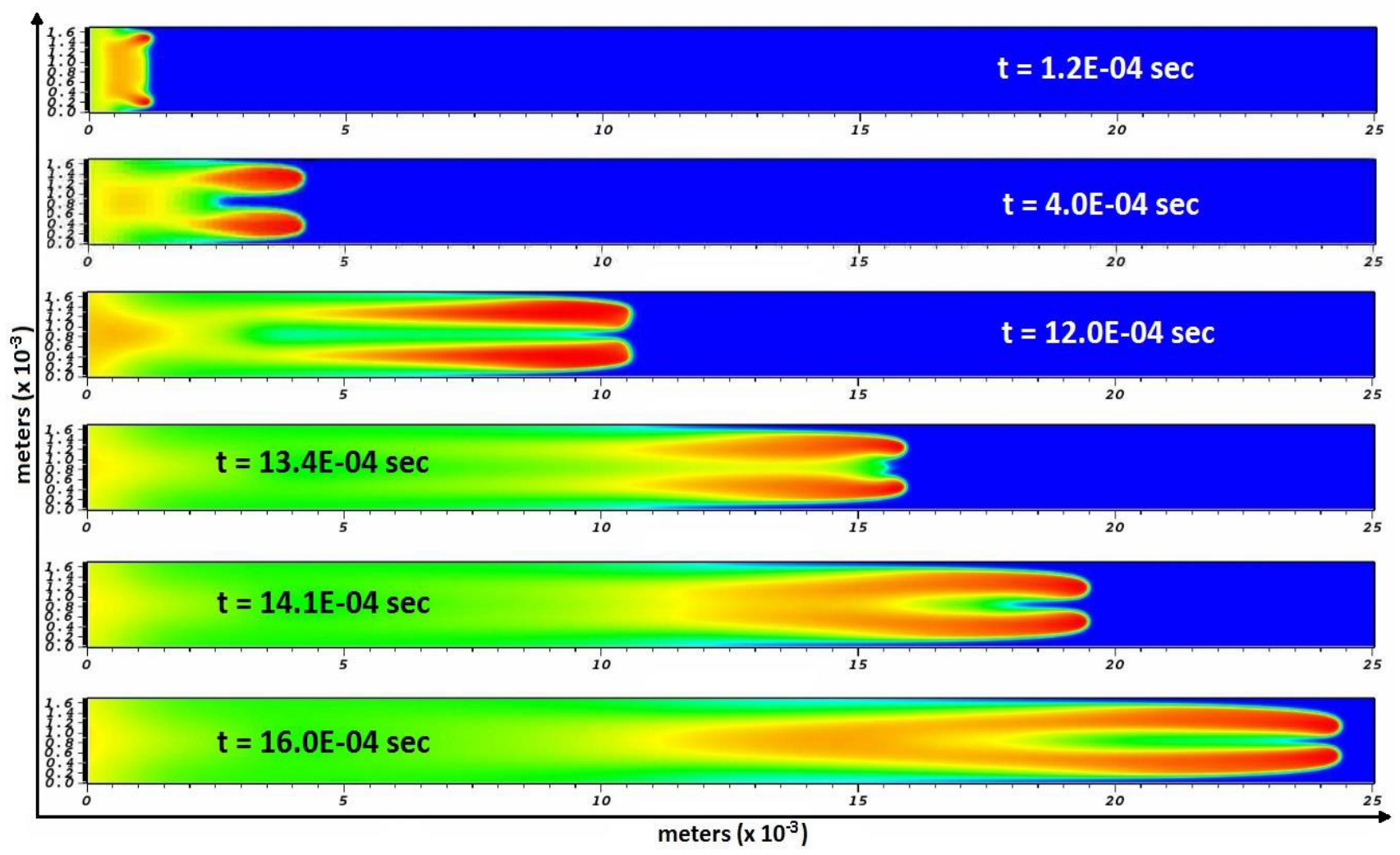

Figure 4.12: Evolution of the central trough for non-equidiffusive flames $(R e=20, L e=0.2)$. 


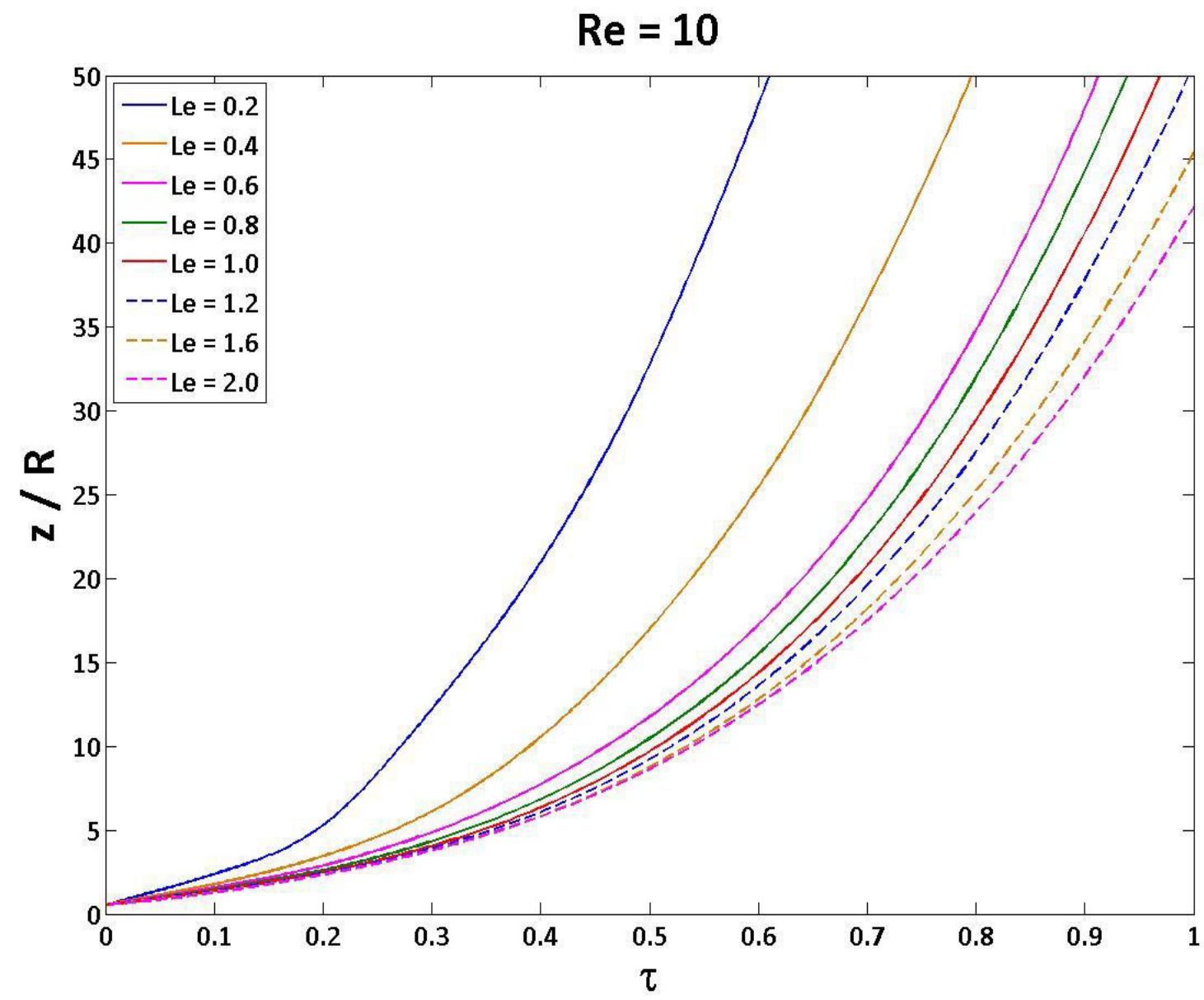

Figure 4.13: The scaled flame tip position $(z / R)$ versus the scaled time $\left(\tau=t U_{f} / R\right)$, for the fixed $R e=10$ $\left(R=20 L_{f}\right)$ and various $L e$ values.

So far, the simulation results undoubtedly showed that the flame splitting into two or more elongated crests (the so-called channeling) greatly increases the flame surface area, and the flame accelerates faster when $L e$ decreases, and vice versa. The flame tip position for various cases is shown in Fig. 4.13, where we observe a negative correlation between $L e$ and the acceleration rate, hence justifying the previous statement. Now, at this point, it is natural to ask what the scenario afterwards is, namely what fate does the split flame will have. To answer this question, namely, in order to observe the behavior of a trough and the crest formation as time passes in the long run, simulations were run for thinner channels, as well (see Fig. 4.14).

Eventually, the simulations for thin channels clearly sketched the picture for the multiple "stages" of the acceleration scenario for non-equidiffusive flames. According to this picture, such stages could be summarized as follows: 
- First stage: If the Lewis number is below a certain critical value, $L e<L e_{C}$, then the trough formation on the flame front grows deeper and flame is literally split in half. Such a case escalates the positive correlation between the flame surface area, and the total burning rate, hence eventually promotes the flame acceleration.

- Second stage: Split flames enter an enhanced acceleration regime where the central flame front is retarded as compared to the upper and lower crests, making the trough deeper and deeper. Eventually, the flame surface area and the burning rate reaches a local maximum value. However, mass diffusion causes the central trough to be devoured by crests at an extremely rapid rate, resulting in the previously "retarded" central flame front to "catch-up" with the crests. This behavior naturally reduces the flame surface area, and moderates the flame momentarily.

- Third stage: As the "retarded" central flame front catches-up with the upper and lower crests, its momentum continues to push the front forward even though the flame slows down due to the rapid decrease in the burning rate. Thus, formation of a third crest at the central axis is observed for $R e>20$. Otherwise, "catching-up" period does not occur "fast enough" for thin tubes, and the central front does not carry enough momentum to push the front and the third crest is not created. Hence, the flame evolution directly enters to the next stage.

- Forth stage: As the trough is mostly (but not fully) consumed, the flame front now continues to accelerate as a whole. If exists, the third crest is also consumed by the upper and lower crests, as observed in the present simulation interval $(R e=10-35)$. One of the interesting outcomes that was apparent in the simulations was the eventual domination of the upper crest in the long run, which can be seen via the snapshots in Fig. 4.14. According to these snapshots, the upper crest surpasses the lower one and the flame front, once again, assumes the finger-like shape, and continues the acceleration period. To be specific, the snapshots of Fig. 4.14 represent the first stage, where the flame is split in half; and the second stage, where the central trough is consumed. The third crest does not form in such narrow tube, and eventually the flame enters the fourth stage where upper crest surpasses the lower, and the flame gets globally finger-like again. 


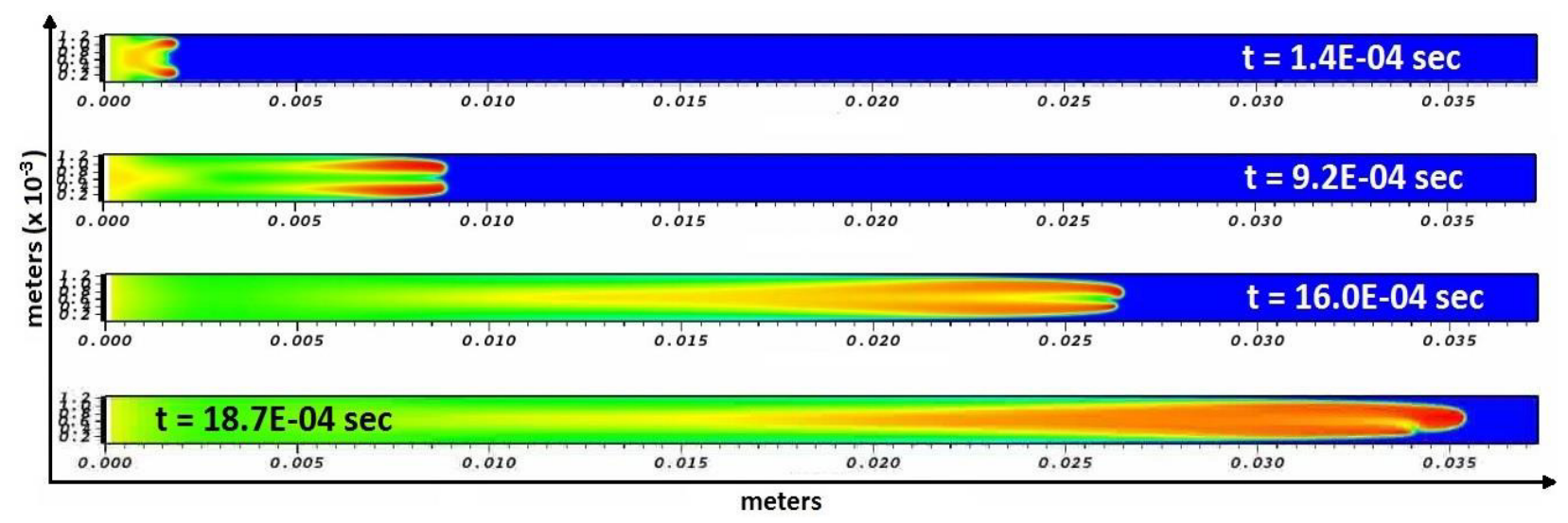

Figure 4.14: Evolution of the "stages" of non-equidiffusive flames $(R e=15, L e=0.2)$.

Another way to analyze these stages is to check the time dependence of the scaled flame tip velocity. Figure 4.15 presents the simulations results for various Lewis number cases, namely $L e$ $=0.2 \sim 1.0$, at fixed $R e=20$. Previously defined stages are apparent on curves' behavior; first as a slight decrease due to the retardation of the center as the central trough gets deeper, and then it is followed by a peak due to the "catch-up" period of the central front. Thereafter, the overall velocity decreases due to the sharp decrease in the total burning rate. It is also observed that the flame front acceleration sharply increases with the decrease in $L e$, i.e. the increase in mass diffusion. Similarly, one can evaluate the scaled burning rate which is directly proportional to the flame surface area (or the flame front length in a 2-D channel), namely $U_{w} / U_{f}=D_{f} / 2 R$, where $D_{f}$ is the length of the flame front. Such an evaluation of the total burning rate could be seen in Fig. 4.16, from simulation results for various Reynolds numbers, namely, $R e=10 \sim 35$, at fixed $L e=0.2$. The stages of the crest and trough formation are all apparent in all curves, except for the $R e=10$ case, where the trough formation is not allowed to become too deep before it is consumed by the crests. Hence, this value can be counted as the threshold below which trough formation is not present. 


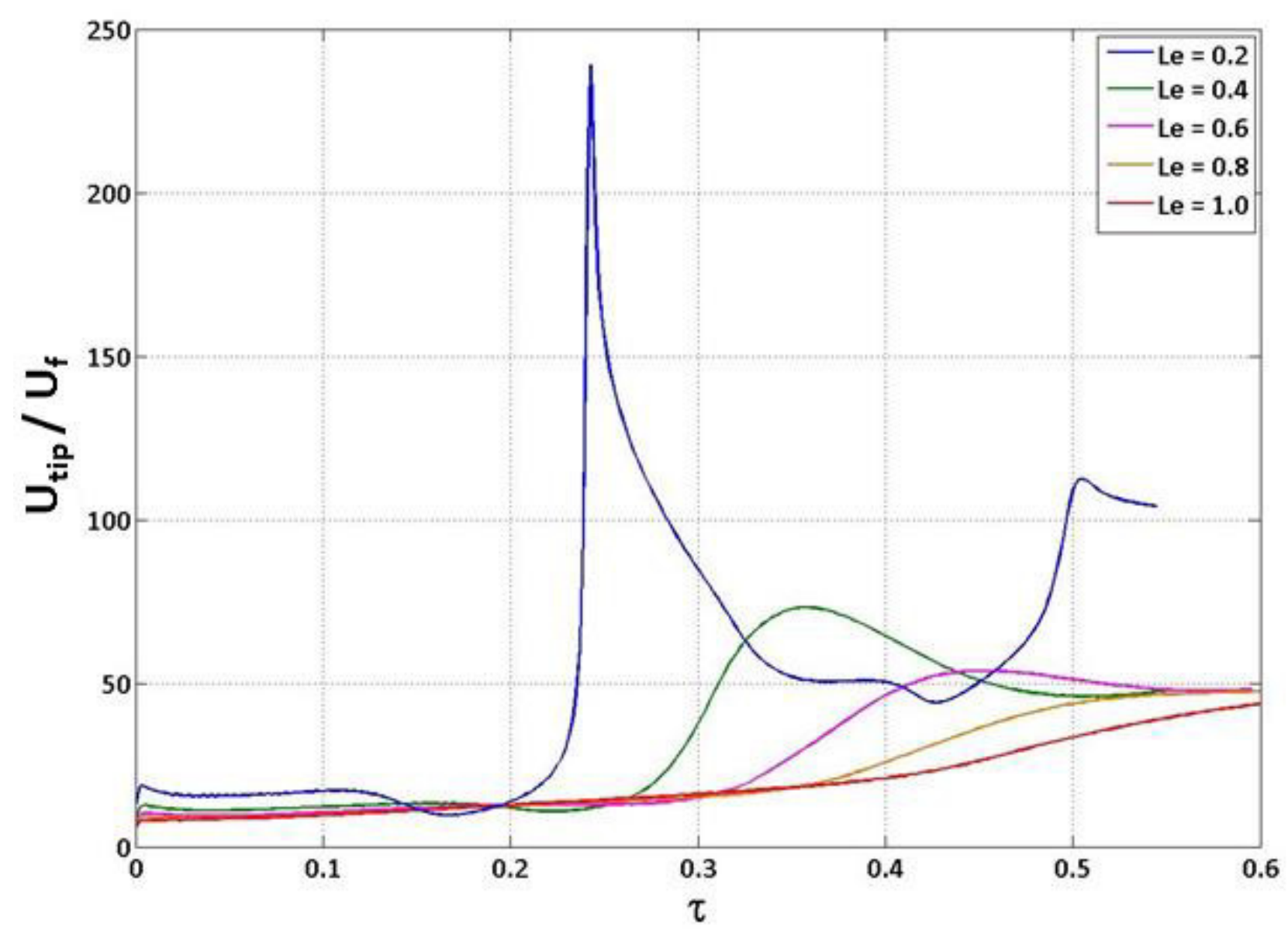

Figure 4.15: The scaled flame tip velocity versus the scaled time $\left(\tau=t U_{f} / R\right)$ for fixed $R e=20\left(R=40 L_{f}\right)$ and various $L e$.

In conclusion, the acceleration scenario of non-equidiffusive flames has been identified in terms of multiple stages. Now, one can discuss the stability conditions for the flame front. Such a trough formation was previously thought to be the result of the initial conditions, or a footprint of the combustion instability, say the $D L$ instability mode. However, by modifying the mass and thermal diffusivity of the fuel, it is seen that the flame surface might promote such an effect in an extensive scale. Therefore, one might also consider that troughs and crests would be a footprint of the $D T$ instability. Such instability is controlled by the activation energy of the fuel, and diffusive properties, which is described by the $M k$ number. It is recalled that the Zel'dovich number was taken in the simulations as small as $Z e=3.5$ for $\Theta=8$, which corresponds to $M k=$ 1.6-1.7 at its minimum. Hence, having $M k>0$ indicates that the flame front is supposed to be stable against the $D T$ instability. Nonetheless, one can define a stability threshold at least for trough development, i.e. $L e_{C}$, which indentifies the boundary separating the two limits: 1) a 
trough appears, yet is quickly consumed before it gets deeper; 2) a trough appears, and continues to get deeper due to mass diffusion effects. Critical $L e$ values for various $R e$-cases are shown in Table 1, which indicates a non-linear yet a positive correlation between the flame split and the channel size. Fig. 4.17 shows an exponential curve fit for these values, whose coefficient of determination $\left(R^{2}\right)$ value is the greatest compared to the other non-linear regression models that have been tried. Yet, the exponential model remains purely observational within the context of this study.

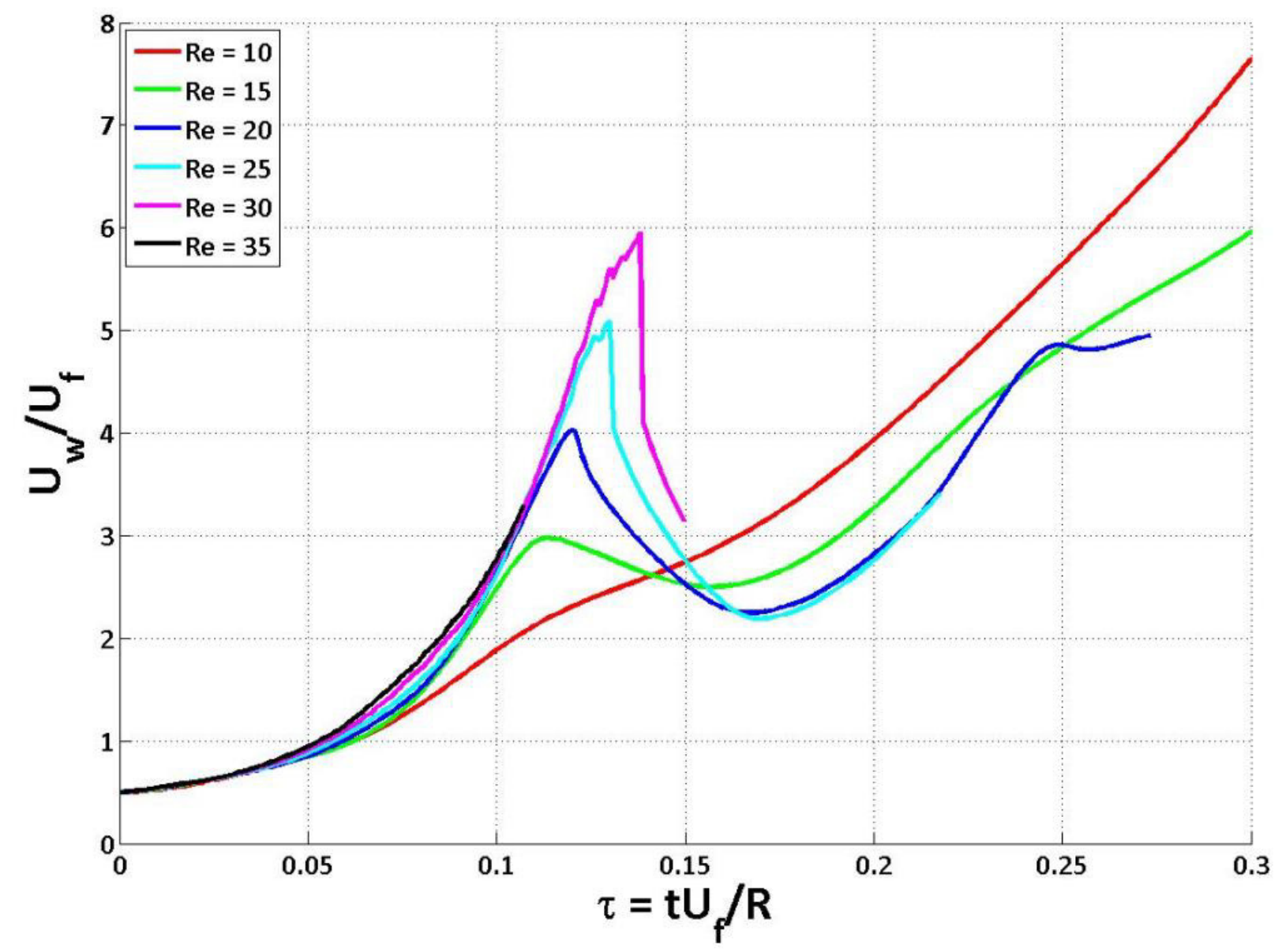

Figure 4.16: The scaled burning rate $\left(U_{w} / U_{f}\right)$ versus the scaled time $\left(\tau=t U_{f} / R\right)$, for the fixed $L e=0.2$, and various $R e=10 \sim 35$.

One can observe sample instances for stable and unstable cases in Fig. 4.18a and 4.18b, respectively. The development of trough is clearly pictured, where it is promptly consumed in stable case, and gets deeper for unstable case. 


\begin{tabular}{|c|c|c|c|c|}
\hline $\boldsymbol{R e}$ & 10 & 15 & 20 & 25 \\
\hline $\boldsymbol{L e}_{\boldsymbol{C}}$ & 0.5 & 0.9 & 1.7 & 2.8 \\
\hline
\end{tabular}

Table 4.1: The critical Lewis number as a stability limit of trough formation, listed for various $R e$ values.



Figure 4.17: $L e_{C}$ versus $R e$ (see Table 1), where the exponential regression model is fitted with the coefficient of determination value of $\mathrm{R}^{2}=0.998$.

However, one should note that the unstable domain defined through $L e_{C}$ is in fact a footprint of pseudo-instability, because even though the crest and trough formation is developed and the flame is split in half, the crests eventually consume the trough and flame front assumes a globally convex shape again, in the long run. However, trough formation substantially increases the overall surface area and the reaction rate, therefore it is an important contributor for the overall flame acceleration. 
a)

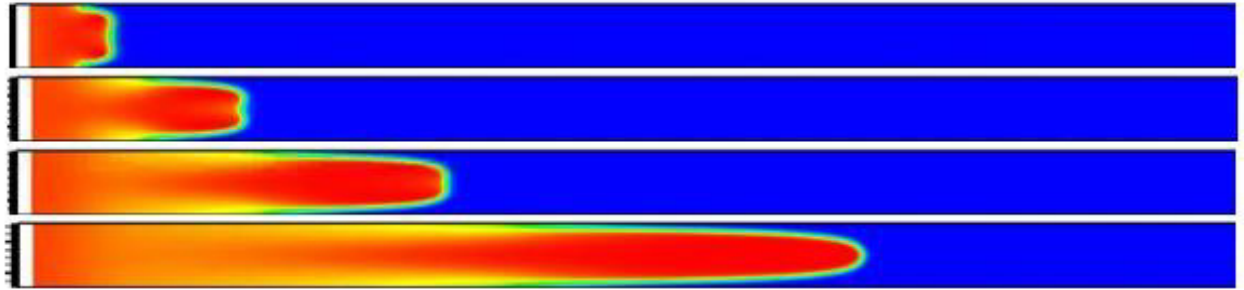

b)
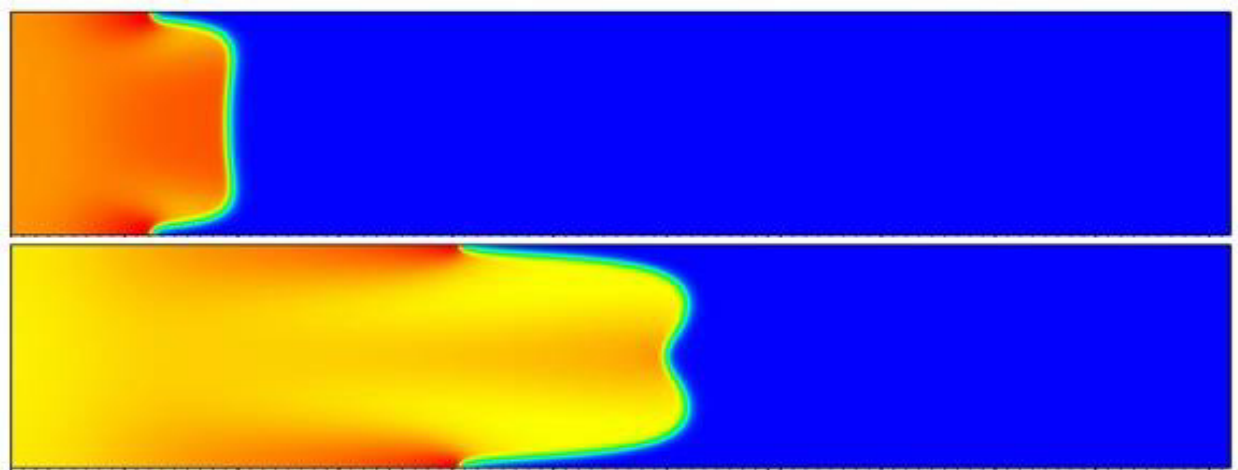

Figure 4.18: a) Evolution of the trough instability for the case when it is suppressed, i.e. $L e>L e_{C}(R e=10, L e=0.6)$,

b) Evolution of the trough instability for the case it develops, i.e. $L e<L e_{C}(R e=25, L e=2.0)$. 


\section{Chapter 5: Analytical Formulations}

In this chapter, ad-hoc theoretical analyses are developed in order to describe and elucidate the simulation results presented in Ch. 4 . The analysis is based on the extension of the Landau-limit formulation of [17] to incorporate the finite thickness of the burning zone by means of $M k$, and thereby Le numbers. Two approaches are used, namely: (i) one derived from the assumption of self-similar flame acceleration; and (ii) another based on a model equation developed from a modified Matalon \& Matkowsky formulation [38].

\subsection{Self-similar Consideration.}

To briefly recall, the analytical theory [17] considered a laminar flame propagating in a two-dimensional (2D) semi-open channel of half-width $R$ with adiabatic and nonslip wall conditions. In the theory, the stream ahead of the flame is approximated by a plane-parallel flow along the walls having the velocity profile $\boldsymbol{u}=\hat{\boldsymbol{e}}_{\mathrm{z}} \mathrm{u}_{\mathrm{z}}(\mathrm{x}, \mathrm{t})$. To simplify the calculations, the conventional scaling is in terms of the channel half-width, $R$, and the unstretched laminar flame velocity, $U_{f}$, such that $(\eta ; \xi)=(x ; z) / R, \tau=t U_{f} / R, \boldsymbol{w}=\boldsymbol{u} / U_{f}$. Then, solving the Navier-Stokes equation for the plane-parallel flow ahead of the flame front, along with nonslip boundary conditions, the following flame evolution equation has been obtained [17]:

$$
-\frac{\partial f}{\partial \tau}=w_{z}(0, \tau)+1-w_{z}-\sqrt{1+\left(\frac{\partial f}{\partial \eta}\right)^{2}},
$$

where the function $f(\eta, \tau)$ describes the flame shape; it is scaled by the half-width of the channel, $R$. The scaled local flame coordinate is then given by $\xi_{f}(\eta, \tau)=\xi_{f}(0, \tau)+f(\eta, \tau)$, with $f(0, \tau)=0$ by the definition. The flame front is driven / distorted by two aspects: one being the propagation with respect to the fuel mixture, and the other is the drifting caused by the flow. Here, the flat top of the flame at the axis moves with the scaled velocity $w_{z}(0, \tau)+1$, whereas the front is also drifted by the flow, which yields the local propagation velocity along the walls as $w_{z}+[1+(\partial f)$ $\left.\partial \eta)^{2}\right]^{1 / 2}$. The last term in the local propagation velocity represents the contribution of the local increase in the flame surface area, which results in flame devouring more fuel mixture per unit time. As a result, Eq. (5.1) indicates how the flame shape gets distorted in time. Under the condition of a strongly inclined front, $|\partial f / \partial \eta| \gg>1$, Eq. (5.1) reduces to

$$
-\frac{\partial f}{\partial \tau}=w_{z}(0, \tau)-w_{z}+\frac{\partial f}{\partial \eta}
$$


for the domain $\eta>0$, since the problem is symmetric with respect to the axis $\eta=0$. Equation (5.2) is linear and has the solution in the form of an exponential acceleration in time:

$$
f(\eta, \tau)=\Phi(\eta) \exp \left(\sigma_{0} \tau\right)
$$

with the acceleration rate assuming the following analytical form (see [17] for the details):

$$
\sigma_{0}=\frac{(R e-1)^{2}}{4 R e}\left(\sqrt{1+\frac{4 R e \Theta}{(R e-1)^{2}}}-1\right)^{2}
$$

To incorporate the effect of a finite flame thickness into this formulation, it is recalled that the viscous effects will be characterized by the Reynolds number associated with the laminar flame propagation, $R e=R U_{f} / v=R / \operatorname{Pr} L_{f}$, where $L_{f}=D_{t h} / U_{f}$ is the conventional definition of the flame thickness, Eq. (3.7). We next employ the classical approach of Matalon \& Matkowsky [38], where the structure of the flame is conventionally approximated as a very thin zone, in which the chemical reactions and active transport processes occur. Matalon \& Matkowsky [38] have derived the equations for the evolution of the shape and location of the flame front, along with the appropriate jump conditions across the front. Using their approach, the equation for the flame stretch term is defined as:

$$
\kappa=\frac{1}{\Delta} \frac{d \Delta}{d \tau}
$$

where $\Delta$ is the scaled surface area of an infinitesimal element on the flame front. In the present limit of a strongly elongated front that accelerates exponentially, one has $\Delta \propto \exp (\sigma \tau)$, which then yields $\kappa=\sigma$. Next, the scaled flame speed definition is employed [38]:

$$
U_{l} / U_{f}=1-\kappa\left(L_{M} / R\right)=1-\sigma M k L_{f} / R
$$

where $L_{M}=M k L_{f}$ is the Markstein length, playing the role of an effective flame thickness with respect to the flame curvature and stretch. Then, one finds the local flame propagation speed $U_{l}$ corrected by the effects of finite flame thickness as

$$
U_{l}=U_{f}\left(1-\sigma M k L_{f} / R\right)=U_{f}(1-\sigma M k / \operatorname{PrRe})
$$

In the limit of a strongly elongated flame front, employed herein, the local flame speed is also a constant. Hence, one may replace the planar flame speed, $U_{f}$, by a modified local flame speed, $U_{l}$, with the cumulative result for the total burning rate being 


$$
U_{w} \propto \exp \left(\frac{\sigma U_{f} t}{R}\right)=\exp \left(\frac{\sigma_{0} U_{l} t}{R}\right)=\exp \left[\sigma_{0}\left(1-\frac{\sigma M k L_{f}}{R}\right) \frac{U_{f} t}{R}\right]=\left[\sigma_{0}\left(1-\frac{\sigma M k}{\operatorname{PrRe}}\right) \tau\right]
$$

As a result, one arrives to a new, modified acceleration rate, $\sigma$, in terms of $\sigma_{0}$, as

$$
\sigma=\sigma_{0}(1-\sigma \mathrm{Mk} / \operatorname{PrRe}) \quad=\quad \sigma=\frac{\sigma_{0}}{1+\sigma_{0} M k / \operatorname{Pr} R e}
$$

with $\sigma_{0}(R e)$ given by [17]; see Eq. (5.4). For simplicity, the Prandtl number is fixed as $\operatorname{Pr}=1$ hereafter, similar to that used in the computational simulations described in Ch. 3 and 4.

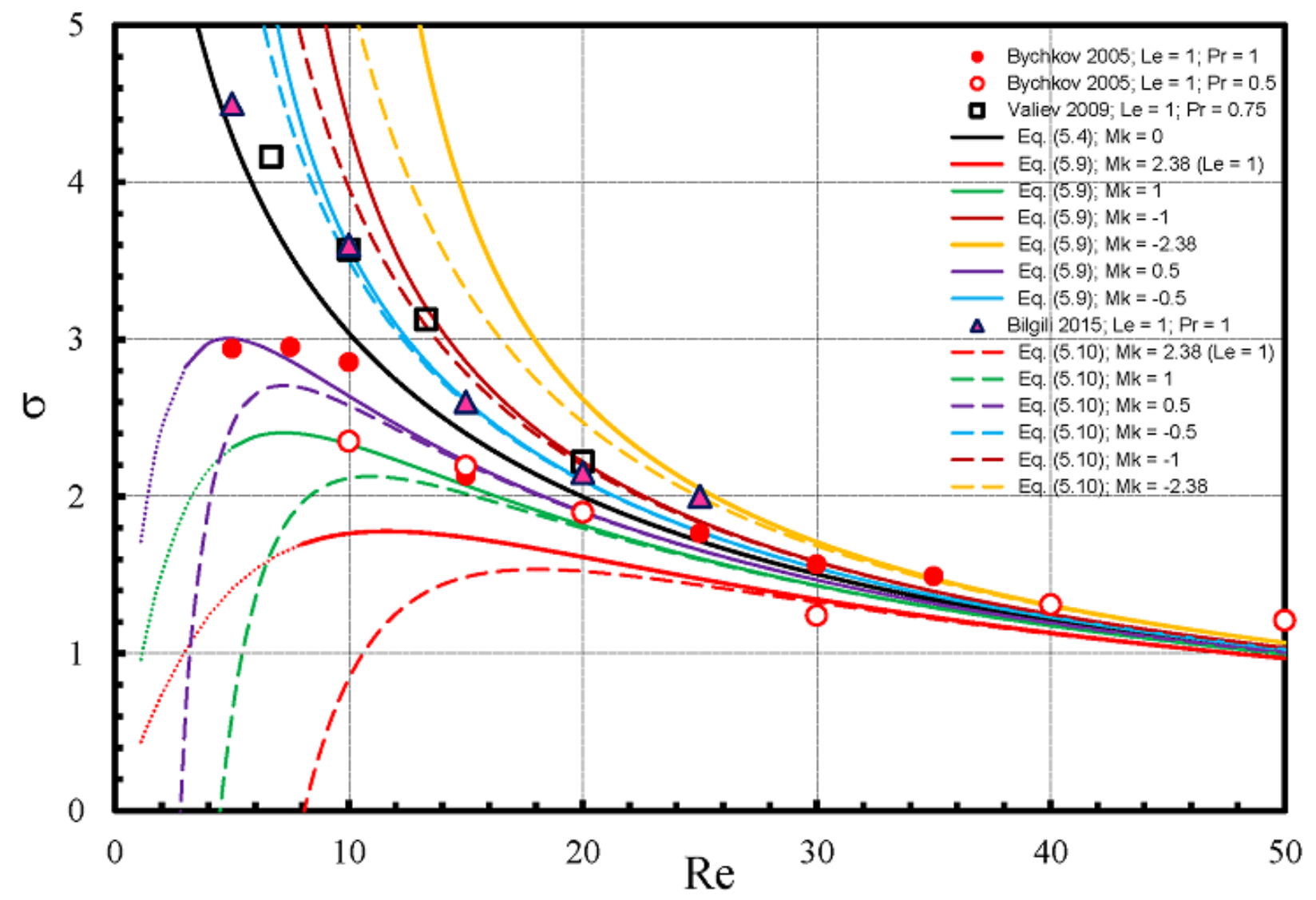

Figure 5.1: Exponential flame acceleration rate $\sigma$ versus the flame propagation Reynolds number, $R e$, for various Markstein numbers, $M k=0 ; \pm 0.5 ; \pm 1 ; \pm 2.38$. Equations (5.9) and (5.10) are presented by the solid/dotted and dashed lines, respectively. The dotted portions denote the break of the theory. Markers show the present simulations, triangles, as well as the previous ones, [17], circles, and [24], squares.

The flame acceleration rate $\sigma$, Eq. (5.9), versus the flame propagation Reynolds number $R e$ is shown in Fig. 5.1 by the solid/dotted lines for a set of fixed $M k=0 ; \pm 0.5 ; \pm 1$; and \pm 2.38 represented by various colors each as specified in the legend. The choice of $M k=2.38$ is related to equidiffusive burning ( $L e=1$ ), according to Eq. (2.2) with $h(\vartheta)=1$, while the case of $M k=0$ reproduces the zero-flame-thickness formulation [17]; see Eq. (5.4). For comparison, a variety of 
numerical simulations on $L e=1$ flames are presented in Fig. 5.1 by the markers. It is seen that the flame stretch may either promote or moderate the flame acceleration depending on the sign of the quantity $M k$. The stretch effect obviously diminishes in wide channels such that all the curves of Fig. 5.1 tend to Eq. (5.4) in the limit of $R e>>1$. A bending of the $M k>0$ curves in Fig. 5.1, at small Re, also has a simple physical explanation: a flame front gets "effectively" thicker with the increase in $M k$, therefore, it is harder to corrugate such a flame at small scales. Consequently, the increase in the flame surface area and thereby the burning rate diminishes such that the acceleration rate decreases. This effect is negligible at large $R e$, when small scales are of minor importance, but it becomes dominant in narrow channels. It is recalled that such a low-Re bending was also observed in the numerical simulations for $L e>1$ flames, see Fig. 4.10, which justifies Eq. (5.9), at least qualitatively.

Nevertheless, the intrinsic limitations of Eq. (5.9) are realized. First of all, it is definitely limited to $\sigma M k / \operatorname{PrRe}<1$ as Eqs. (5.7) would formally yield a "negative" flame velocity $U_{l}$ otherwise. The dotted "tails" of the curves in Fig. 5.1 correspond to such an unphysical situation. Hence, only the solid parts of the curves are of relevance. To be more rigorous, this limitation should be extended to $\sigma M k / \operatorname{PrRe} \ll 1$, because Eq. (5.6) is actually a first-order expansion in a small parameter $R e^{-1}$, with the higher order terms, $O\left(R e^{-2}\right)$, omitted. In this respect, let us propose an alternative of Eq. (5.9) in the form

$$
\sigma=\sigma_{0}\left(1-\sigma_{0} M k / \operatorname{Pr} R e\right)
$$

The rational is the following: Eqs. (5.9) and (5.10) differ only by the $2^{\text {nd }}$ and higher order terms in $R e^{-1}$. Consequently, both equations should coincide within the validity domain of Eq. (5.6). Equation (5.10) is shown in Fig. 5.1 by the dashed lines, with various $M k$ represented by the same color as in the solid/dotted lines. The $R e$ value, at which the solid and dashed lines of a given color start deviating, denotes the lower limit of the accuracy of formulations (5.9) - (5.10) for the $M k$ quantity associated with this color. It is seen from Fig. 5.1 that for most $M k$ from the practical reality the present formulation start deteriorating for $R e<10 \sim 15$, and it completely breaks for $R e<5 \sim 10$ with $M k>0$ (dashed parts). Consequently, at small $R e$, Eqs. (5.9) and (5.10) may work only as models, keeping in mind that the most intriguing effect of flame thickening occurs at these scales. In fact, one may not anticipate a brilliant outcome from the combination of the studies [38] and [17] anyway, since Matalon \& Matkowsky [38] dealt with 
the approach of weak corrugation and weak stretch while Bychkov and co-authors [17] deployed the opposite limit.

\begin{tabular}{|l|l|l|l|l|l|l|l|c|}
\hline$L e$ & 0.2 & 0.4 & 0.6 & 0.8 & 1.0 & 1.2 & 1.6 & 2 \\
\hline$M k$ & 1.7 & 1.87 & 2.03 & 2.21 & 2.38 & 2.55 & 2.89 & 3.22 \\
\hline
\end{tabular}

Table 5.1: Coupling between the Markstein, $M k$, and Lewis, $L e$, numbers according to Eq. (2.2) with $h(\vartheta)=1$.

For quantitative comparison between the simulations of Ch. 4 and Eqs. (5.9), (5.10), one should tabulate the latter in terms of $L e$ instead of $M k$. This is undertaken in Fig. 5.2, with the relationship between $M k$ and $L e$ calculated by Eq. (2.2), with $h(\vartheta)=1$, and presented in Table 5.1.

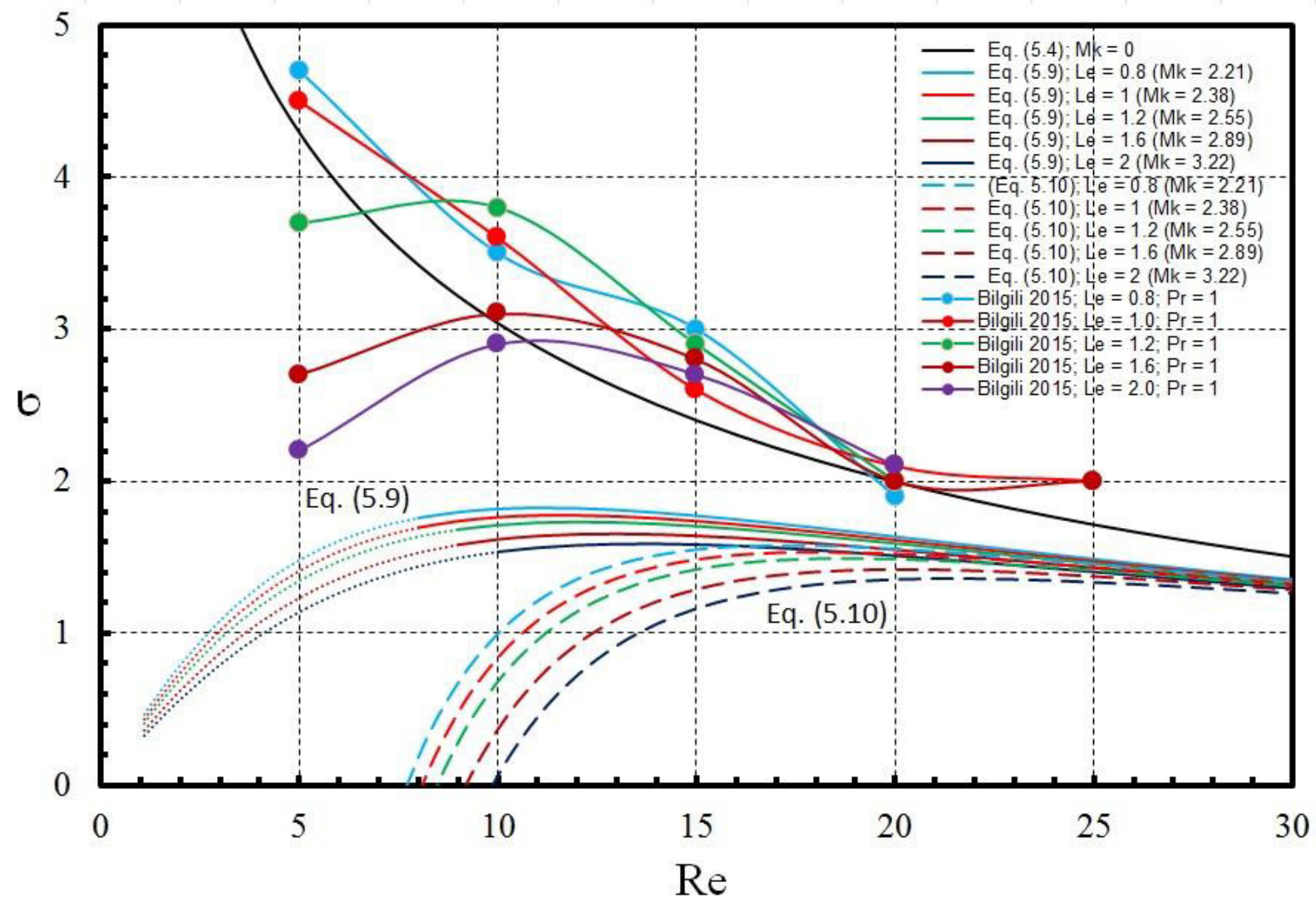

Figure 5.2: Exponential flame acceleration rate $\sigma$ versus the flame propagation Reynolds number, $R e$, for various Lewis numbers, $L e=0.8 ; 1 ; 1.2 ; 1.6 ; 2.0$. Equations (5.9) and (5.10) are presented by the solid/dotted and dashed lines, respectively. The dotted portions denote the break of the theory. The black solid line is related to $M k=0$, Eq. (5.4). Markers show the present simulations. Overall, the same colors correspond to the same Le numbers.

Again, the dashed lines in Fig. 5.2 present Eq. (5.10), while Eq. (5.9) is shown by the solid lines, with dotted "tails" associated with an unphysical situation of a formally negative flame speed. Deviations between the dashed and solid lines of the same color yield the low-Re 
limitations of the analytical theory for a given $L e$ at small $R e$. For $R e>>1$ both Eqs. (5.9) and (5.10) coincide and tend to the limit (5.4). The cloud of markers fully reproduces that of Fig. 4.10.

The dependence of $\sigma$ versus $L e$ at fixed $R e$ has also been scrutinized. The result is shown in Fig. 5.3, for $R e=5$ (Fig. 5.3a), $R e=10$ (Fig. 5.3b), $R e=15$ (Fig. 5.3c) and $R e=20$ (Fig. 5.3d), respectively. For illustrative purposes, the linear trend of the simulation results is also presented in all the plots, which is helpful when compared with the analytical endeavors, Eqs. (5.9), (5.10).
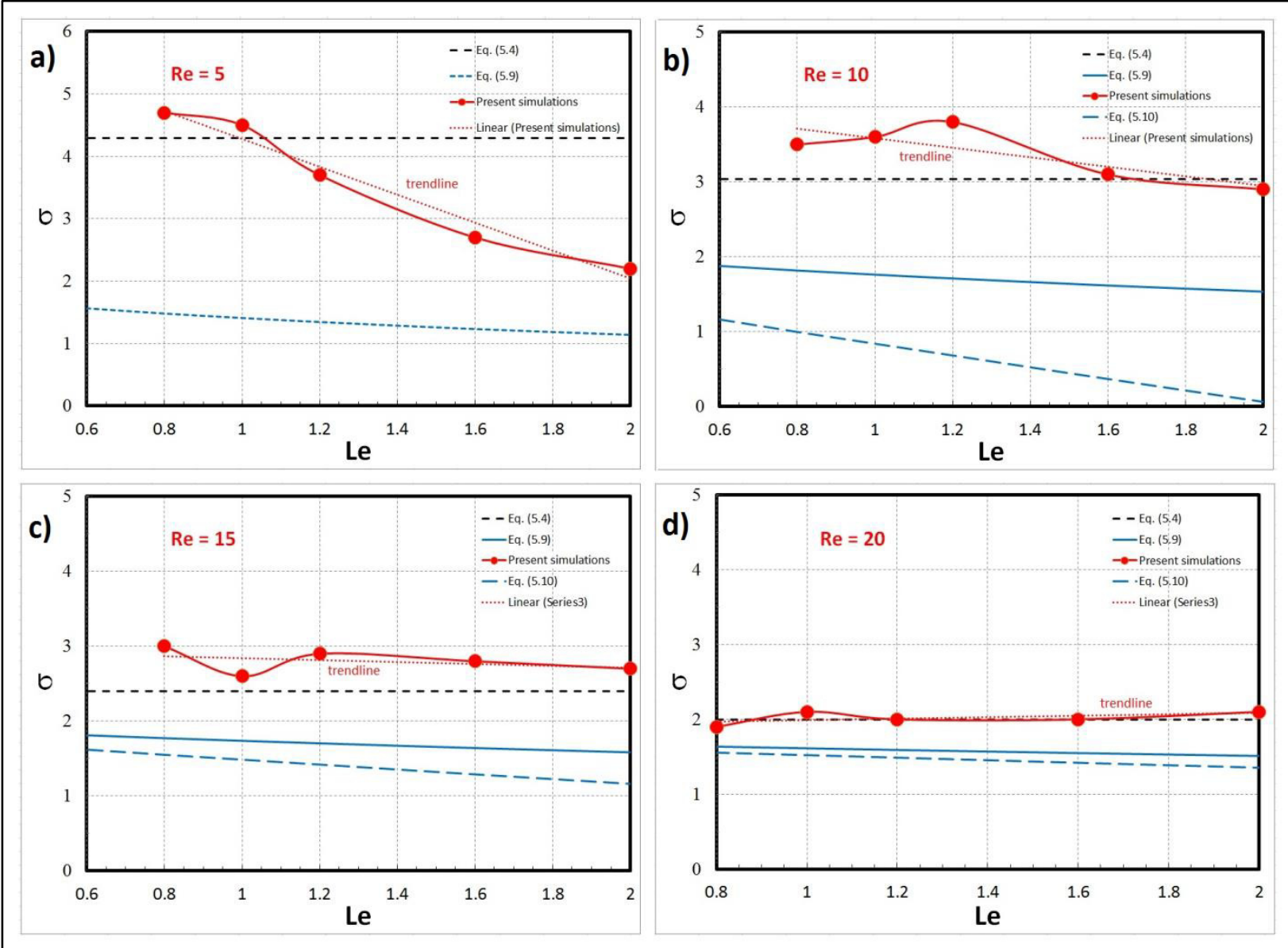

Figure 5.3: Exponential flame acceleration rate $\sigma$ versus the Lewis number, $L e$, for various fixed flame propagation Reynolds numbers: $R e=5$ (a); 10 (b); 15 (c) and 20 (d). In all the plots, Eqs. (5.9) and (5.10) are presented by the blue solid/dotted and dashed lines, respectively. The dotted black lines show Eq. (5.4). The present simulations are shown by markers, with the linear trend presented by the red totted line.

One can conclude from Figs. 5.2 and 5.3 (a-d) that Eq. (5.9) agrees qualitatively with the simulation results in terms of two major trends. First, a bending trend (the reduction in the acceleration rate) at small $R e$, associated with the flame thickening, is observed both in the 
modeling and theory. Second, both the simulations and the theory show a decrease in $\sigma$ experienced both with the increase in $L e$ and in $R e$. Still, there is no good quantitative agreement between the theory and modeling, which was anticipating accounting the limitations of the theory mentioned above. Let us also point out a conceptual difference between the theory and modeling: The simulation results change drastically with the variations of the Lewis number. Namely, the simulations show almost monotonic $R e$-dependences for $L e=0.8,1.0$, resembling, strikingly, the $M k=0$ result (5.4). In contrast, a bending trend, related to the flame thickening, is already detected in the simulations for $L e \geq 1.2$. Thus, only a slight change in $M k$ and $L e$ leads to appearance of such a significant effect in the present modeling. In contrast, all the theoretical curves for $L e=0.8 \sim 2.0$, go very close to each other, and they deviate considerably from Eq. (5.4).

In order to extend the accuracy and validity of the present formulation, and to reduce the existing quantitative gap between the theory and modeling, let us employ the following model modification: When defining the Markstein length as $L_{M}=M k L_{f}$, one should remember that the flame "thickness" $L_{f}$ is actually a useful mathematical parameter of length dimension, which however is less than the thermal flame zone, but exceeds the active reaction zone in the reality. In this respect, why not replace $M k$ with its re-defined version, namely the effective Markstein number, $M k_{e f f}$ ? Then for the counterpart of Eq. (5.9), one arrives to a new modified acceleration rate, $\sigma$, in terms of $\sigma_{0}$, as

$$
\sigma=\frac{\sigma_{0}}{1+\sigma_{0} M k_{e f f} / \operatorname{Pr} R e},
$$

with its "conjugative" - analogue of Eq. (5.10) being

$$
\sigma=\sigma_{0}\left(1-\frac{\sigma_{0} M k_{e f f}}{\operatorname{PrRe}}\right)
$$

Without having a rigorous idea of what $M k_{e f f}$ is, one may guess that is should depend on the thermal-chemical flame properties, such as (non)-equidiffusivity and thermal expansion, but not on the flow properties. Therefore, it could be assumed that $M k_{e f f}$ is $R e$-independent, but involves a functional dependence on $M k$ itself. Besides, $M k_{\text {eff }}$ overall should be, say, of the order of $10^{-1}$ in order to justify the $1^{\text {st }}$ - order expansion in $\sigma_{0} M k_{e f f} / \operatorname{Pr} R e \ll 1$ for small and 
moderate $\operatorname{Re}, \operatorname{Re}=5-20$. Keeping all these hints in mind, and aiming to fit the simulation result as reasonable as possible, the following phenomenological formula is proposed:

$$
M k_{e f f}=\Gamma M k^{n},
$$

where $\Gamma$ and $n$ are phenomenological constants. Figures 5.4 and 5.5 are counterparts of Figs. 5.2 and 5.3, with Eqs. (5.11) and (5.12) employed instead of Eqs. (5.9) and (5.10), respectively. The quantity $M k_{e f f}$ was calculated by means of Eq. (5.13), where the arbitrary constants are set to $\Gamma=0.001$ and $n=5.5$. It is clearly seen that the modified formulation yields much better agreement with the simulation results than the original formulation. Indeed, the theoretical curves in Figs. 5.4 and 5.5 reproduce the computational trends not only qualitatively, but also quantitatively - up to a certain extent, of course. One should nevertheless remember that Eqs. (5.11) - (5.13) are just a model; a more rigorous analyses how to quantify and incorporate the factor $M k_{e f f}$ constitutes a subject of a future work.

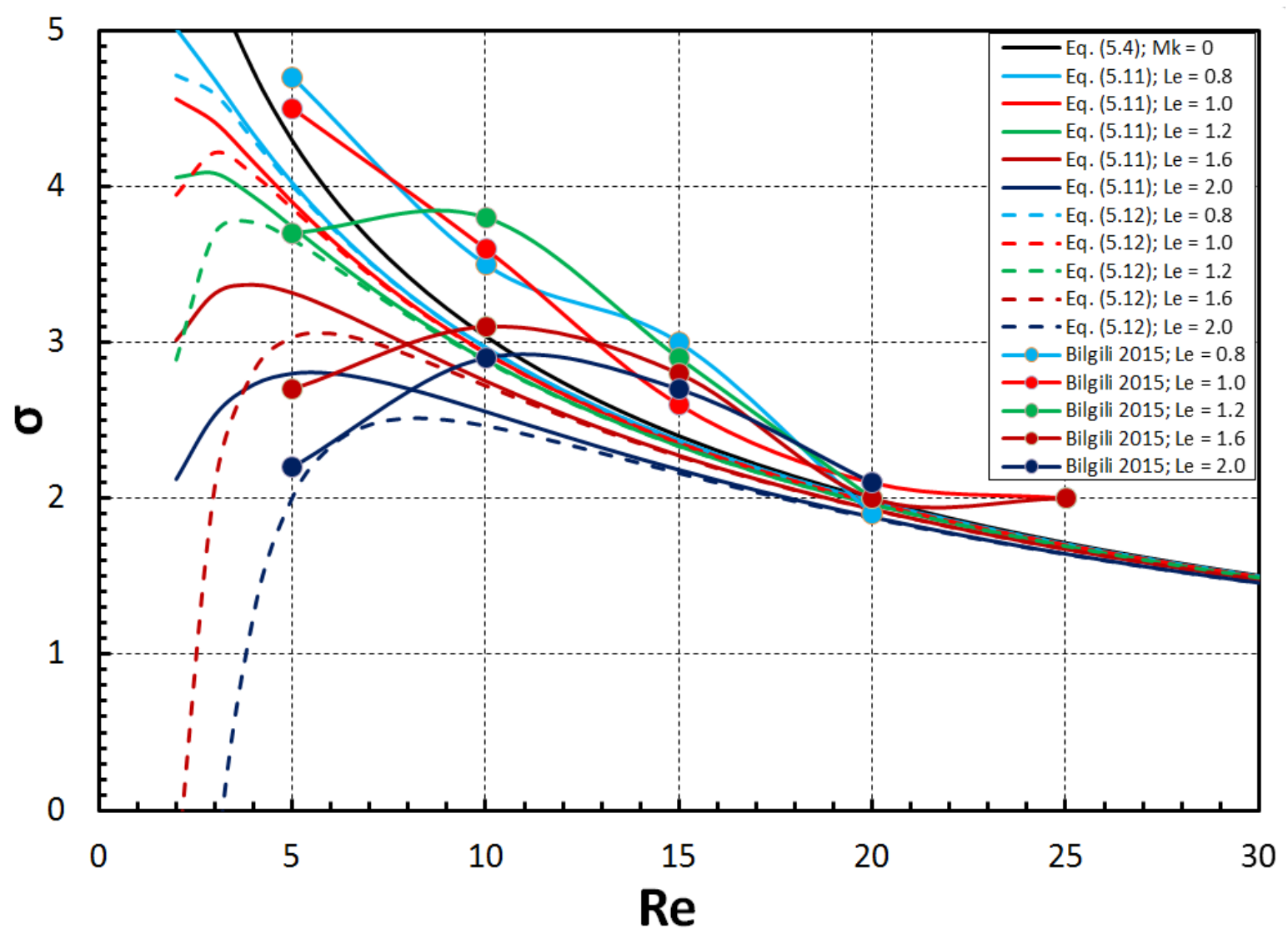

Figure 5.4: Exponential flame acceleration rate $\sigma$ versus the flame propagation Reynolds number, $R e$, for various Lewis numbers, $L e=0.8 ; 1 ; 1.2 ; 1.6 ; 2.0$. Equations $(5.11)$ and $(5.12)$ are presented by the solid/dotted and dashed lines, respectively. The dotted portions denote the break of the theory. The black solid line is related to $M k=0$, Eq. (5.4). Markers show the present simulations. Overall, the same colors correspond to the same Le numbers. The factor $M k_{e f f}$ is given by Eq. (5.13), where $\Gamma=0.001$ and $n=5.5$. 

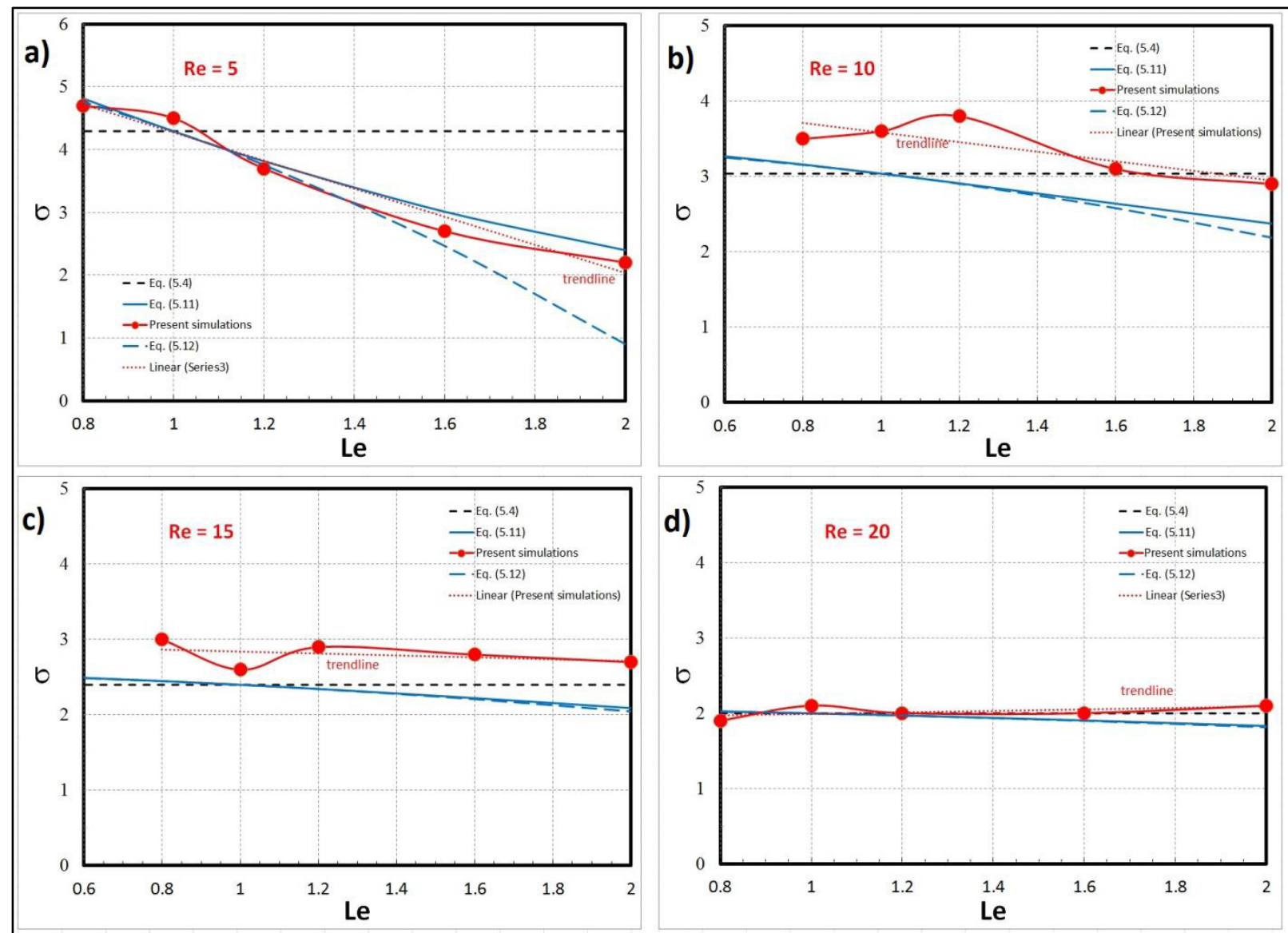

Figure 5.5: Exponential flame acceleration rate $\sigma$ versus the Lewis number, Le, for various fixed flame propagation Reynolds numbers: $R e=5$ (a); 10 (b); 15 (c) and 20 (d). In all the plots, Eqs. (5.11) and (5.12) are presented by the blue solid/dotted and dashed lines, respectively. The dotted black lines show Eq. (5.4). The present simulations are shown by markers, with the liner trend presented by the red dotted line. The factor $M k_{e f f}$ is given by Eq. (5.13), where $\Gamma=0.001$ and $n=5.5$.

\subsection{Alternative Formulation}

In this section, an alternative analytic approach is presented for accounting the $M k$-related effects on the flame acceleration scenario. Firstly, to incorporate the flame thickness, on should recall the classical approach of Matalon \& Matkowsky [38], where the structure of the flame is considered to consist of a boundary layer in which the chemical reactions occur, which is also located inside another boundary layer in which transport processes dominate. The authors of [38] have derived the equations for the evolution of the shape and location of the flame front, along with the appropriate jump conditions across the front. Following their approach, let us consider the equation for the motion of the flame front [38]: 


$$
\begin{aligned}
\{u(y, z, t)-\boldsymbol{v} & \left.(y, z, t) \cdot \nabla f-\frac{\partial f}{\partial t}\right\} N^{-1} \\
& =1-\delta M k\left\{\frac{\nabla^{2} f}{N}+\nabla \cdot v(y, z, t)+\frac{1}{N} \frac{D N}{D t}\right\}+o(\delta),
\end{aligned}
$$

where the scaled increase in the flame surface area is defined as $N=\left(1+|\nabla f|^{2}\right)^{1 / 2}$, and the correction terms in the right hand side are proportional to the scaled flame thickness, $\delta=L_{f} / R$, and $M k$. To adopt and compare Eq. (5.14) along with Eq. (2.1), some adjustments are required to make both formulations compatible, which are elucidated in following sub-sections.

\subsubsection{Plane parallel flow and notation correction}

The velocity field in Eq. (5.14) is defined as $\overrightarrow{\boldsymbol{V}}=u(y, z, t) \hat{\boldsymbol{\imath}}+\overrightarrow{\boldsymbol{v}}$ for a flame propagating in $x$-direction, and $\vec{v}$ is the 2D transverse velocity component vector [38]. Since the assumption of a fully-developed plane-parallel flow is undertaken for a flame propagating in the $z$-direction, one can neglect the transverse component, i.e. $\vec{v}=0$. Also, it is convenient to switch the notation as $t \rightarrow \tau, x \rightarrow \eta, v_{z} \rightarrow w_{z}(\eta, \tau)$, hence $f(x, t) \rightarrow f(\eta, \tau)$. Thus Eq. (5.14) is simplified as follows:

$$
-\frac{\partial f}{\partial \tau}=-w_{z}+N-\epsilon\left(\frac{\partial^{2} f}{\partial \eta^{2}}+\frac{\partial N}{\partial \tau}\right)
$$

where the correction parameter is defined as $\epsilon=\delta M k$.

\subsubsection{Flame function sign correction}

Substituting the parameter $N$ into the Eq. (5.15) yields

$$
-\frac{\partial f}{\partial \tau}=-w_{z}+\left[1+\left(\frac{\partial f}{\partial \eta}\right)^{2}\right]^{1 / 2}-\epsilon\left(\frac{\partial^{2} f}{\partial \eta^{2}}+\frac{\partial}{\partial \tau}\left[1+\left(\frac{\partial f}{\partial \eta}\right)^{2}\right]^{1 / 2}\right)
$$

The flame front function defined in [38] is of the form $\xi_{f}=\xi_{f}(0, \tau)-f(\eta, \tau)$, whereas [17] employs $\xi_{f}=\xi_{f}(0, \tau)+f(\eta, \tau)$. Observing differences in sign convention, it seems necessary and convenient to do a sign correction for consistence with the present formulation, namely $f \rightarrow-f$ as:

$$
\frac{\partial f}{\partial \tau}=-w_{z}+\left[1+\left(-\frac{\partial f}{\partial \eta}\right)^{2}\right]^{1 / 2}-\epsilon\left(-\frac{\partial^{2} f}{\partial \eta^{2}}+\frac{\partial}{\partial \tau}\left[1+\left(-\frac{\partial f}{\partial \eta}\right)^{2}\right]^{1 / 2}\right)
$$

Following the present condition of a strongly inclined front, that is $(\partial f / \partial \eta)>>1$, one can ignore the unity terms in the $N$ term. Hence, Eq. (5.17) takes the form 


$$
\frac{\partial f}{\partial \tau}=-w_{z}+\left|\frac{\partial f}{\partial \eta}\right|-\epsilon\left(-\frac{\partial^{2} f}{\partial \eta^{2}}+\frac{\partial}{\partial \tau}\left|\frac{\partial f}{\partial \eta}\right|\right)
$$

\subsubsection{Reference frame correction}

Exploiting the symmetry of the problem, one can consider the domain $\eta>0$, where ( $\partial f$ ) $\partial \eta)<0$. Hence, Eq. (5.18) becomes

$$
-\frac{\partial f}{\partial \tau}=w_{z}+\frac{\partial f}{\partial \eta}-\epsilon\left(\frac{\partial^{2} f}{\partial \eta^{2}}+\frac{\partial^{2} f}{\partial \tau \partial \eta}\right)
$$

Note that, Eq. (5.19) describes the motion of the flame front within the reference frame attached to the flame itself, as it is constructed in [38]. To switch to the laboratory reference frame, as to be similar to [17], one needs to modify the velocity term. Namely, the velocity should be modified as $w_{z}=w_{z}(0, \tau)-w_{z}+1$. One can therefore rewrite Eq. (5.19) as

$$
-\frac{\partial f}{\partial \tau}=w_{z}(0, \tau)-w_{z}+\frac{\partial f}{\partial \eta}-\epsilon\left(\frac{\partial^{2} f}{\partial \eta^{2}}+\frac{\partial^{2} f}{\partial \tau \partial \eta}\right)
$$

where the unity term in the corrected velocity term is again ignored due to the dominant slope term.

\subsubsection{Curvature term correction}

Another necessary correction is related to the term corresponding to the curvature effects in Eq. (5.20), namely $\partial^{2} f / \partial \eta^{2}$. Considering a typical function $f(x)=y$, the curvature can be defined as $\kappa=\left|y^{\prime \prime}\right| /\left(1+\left(y^{\prime}\right)^{2}\right)^{3 / 2}$. In [38], it was assumed that the slope along the flame tip is quite small compared to the unity, namely $(\partial f / \partial \eta)<<1$. Therefore, the curvature is approximated by $\kappa \cong\left|y^{\prime \prime}\right|$, which is the curvature term in Eq. (5.14), and hence present in the modified evolution equation, Eq. (5.20). However, the reciprocal assumption is undertaken in [17], which deals with a strong distortion in the flame tip, namely $(\partial f / \partial \eta)>>1$. Thus, the curvature becomes $\kappa \cong\left|y^{\prime \prime}\right| /\left(y^{\prime}\right)^{3}$. Substituting the latter approximate curvature term into Eq. (5.20), one attains the final form of the modified evolution equation, where the flame thickness effects are incorporated through correction terms:

$$
-\frac{\partial f}{\partial \tau}=w_{z}(0, \tau)-w_{z}+f^{\prime}-\epsilon\left(\frac{f^{\prime \prime}}{\left(f^{\prime}\right)^{3}}+\frac{\partial}{\partial \tau} f^{\prime}\right),
$$

or, rearranging the terms 


$$
-\frac{\partial}{\partial \tau}\left[f-\epsilon f^{\prime}\right]=w_{z}(0, \tau)-w_{z}+\left[f^{\prime}-\epsilon \frac{f^{\prime \prime}}{\left(f^{\prime}\right)^{3}}\right] .
$$

It is observed that Eq. (5.22) is the counterpart of Eq. (5.2), i.e. ignoring the correction terms (when $\epsilon=0$ ) Eq. (5.22) truncates back to Eq. (5.2).

\subsubsection{Solution for the modified flame evolution equation}

Equation (5.22) is strongly non-linear and it is challenging to split it into the spatial and time components. Recalling the present condition of a strongly inclined front, $(\partial f / \partial \eta)>>1$, it is plausible to state that curvature effects are negligible. Hence Eq. (5.22) reduces to

$$
-\frac{\partial}{\partial \tau}\left[f-\epsilon f^{\prime}\right]=w_{z}(0, \tau)-w_{z}+f^{\prime}
$$

In order to define the velocity profile, one should quote the Navier-Stokes equation obeyed by the plane-parallel flow ahead of the flame front [17]:

$$
\frac{\partial w_{z}}{\partial \tau}=-\frac{\partial p}{\partial \zeta}+\frac{1}{R e} \frac{\partial^{2} w_{z}}{\partial \eta^{2}}
$$

where the pressure gradient is produced by the flame front along the scaled propagation direction, and density and pressure are scaled by $\rho_{f}$ and $\rho_{f} U_{f}{ }^{2}$, respectively [17]. Within the approach of exponential regime for the flame acceleration, the solution for the velocity profile pushed by the flame yields [17]:

$$
w_{z}=-(\Theta-1) f(1, \tau) \frac{\cosh (\mu)-\cosh (\mu \eta)}{\cosh (\mu)-\mu^{-1} \sinh (\mu)},
$$

where $\mu=\sqrt{\sigma R e}$. Similar to Eq. (2.3), a solution in the form of exponential acceleration in time is desired, namely

$$
f(\eta, \tau)=\left[\Phi_{0}(\eta)+\epsilon \Phi_{1}(\eta)\right] \exp (\sigma \tau)
$$

where the acceleration rate incorporating a small but finite flame thickness is defined to the first order correction as $\sigma=\sigma_{0}+\epsilon \sigma_{1}$. Substituting Eq. (5.26) into Eq. (5.25), then Eq. (5.24); and then separating the zeroth and first order terms with respect to the correction factor, $\epsilon$, one obtains the following differential equations for terms with, and without $\epsilon$, respectively:

$$
\Phi_{0}^{\prime}=-\sigma_{0} \Phi_{0}+(\Theta-1) \Phi_{0}(1) \frac{\cosh (\mu \eta)-1}{\cosh (\mu)-\mu^{-1} \sinh (\mu)}
$$




$$
\Phi_{1}{ }^{\prime}=-\sigma_{1} \Phi_{0}-\sigma_{0}\left(\Phi_{1}-\Phi_{0}{ }^{\prime}\right)+(\Theta-1) \Phi_{1}(1) \frac{\cosh (\mu \eta)-1}{\cosh (\mu)-\mu^{-1} \sinh (\mu)}
$$

Equation (5.27) yields the zeroth order flame shape function as

$$
\Phi_{0}(\eta)=\frac{(\Theta-1) \Phi_{0}(1) \exp \left(-\sigma_{0} \eta\right)}{\cosh (\mu)-\mu^{-1} \sinh (\mu)} \int_{0}^{\eta}\left[\cosh \left(\mu \eta^{\prime}\right)-1\right] \exp \left(\sigma_{0} \eta^{\prime}\right) d \eta^{\prime}
$$

which can be integrated as

$$
\begin{aligned}
& \Phi_{0}(\eta)=\frac{(\Theta-1) \Phi_{0}(1)}{\cosh (\mu)-\mu^{-1} \sinh (\mu)}\left[\frac{\exp (\mu \eta)}{2\left(\mu+\sigma_{0}\right)}-\frac{\exp (-\mu \eta)}{2\left(\mu-\sigma_{0}\right)}+\frac{\mu^{2}}{\mu^{2}-\sigma_{0}^{2}} \frac{\exp \left(-\sigma_{0} \eta\right)}{\sigma_{0}}\right. \\
& \left.-\frac{1}{\sigma_{0}}\right]
\end{aligned}
$$

for the region where $\eta>0$, which is already found in [17]. Then, the zeroth order acceleration rate could be found by exploiting the condition $\Phi_{0}(\eta)=\Phi_{0}(1)$ for $\eta=1$

$$
\sigma_{0}=\frac{(R e-1)^{2}}{4 R e}\left(\sqrt{1+\frac{4 R e \Theta}{(\operatorname{Re}-1)^{2}}}-1\right)^{2}
$$

in the limit of large thermal expansion leading to $\mu>>1$. Obviously, Eq. (5.31) is identical to Eq. (5.4), as expected, since the same methodology of [17] is followed. In a similar manner, following a similar approach to integrate Eq. (5.28) results in the first order flame shape function:

$$
\Phi_{1}(\eta)=C\left\{A \Phi_{0}(1)+B \Phi_{1}(1)\right\}
$$

where the parameters $\mathrm{A}, \mathrm{B}$, and $\mathrm{C}$ are defined as

$$
\begin{aligned}
\mathrm{A}=\exp (-\mu \eta) & \left\{\sigma_{0}{ }^{3}-\eta \sigma_{0}{ }^{4}+\mu{\sigma_{0}}^{2}-\eta \mu \sigma_{0}{ }^{3}-\eta \sigma_{0}{ }^{2} \sigma_{1}-\eta \mu \sigma_{0} \sigma_{1}\right\} \\
+ & \exp (\mu \eta)\left\{\sigma_{0}{ }^{3}-\eta \sigma_{0}{ }^{4}-\mu{\sigma_{0}}^{2}+\eta \mu \sigma_{0}{ }^{3}-\eta \sigma_{0}{ }^{2} \sigma_{1}+\eta \mu \sigma_{0} \sigma_{1}\right\} \\
+ & \exp \left(-\sigma_{0} \eta\right)\left\{2 \eta \mu^{2}{\sigma_{0}}^{2}-2 \mu^{2} \sigma_{0}+2 \eta \mu^{2} \sigma_{1}\right\}-2 \sigma_{0}{ }^{3}+2 \eta \sigma_{0}{ }^{4} \\
- & 2 \eta \mu^{2} \sigma_{1}+2 \eta \sigma_{0}{ }^{2} \sigma_{1}-2 \eta \mu^{2}{\sigma_{0}}^{2}+2 \mu^{2} \sigma_{0}, \\
\mathrm{~B}=\exp (-\mu \eta) & \left\{{\sigma_{0}}^{2}+\mu \sigma_{0}\right\}+\exp (\mu \eta)\left\{{\sigma_{0}}^{2}-\mu \sigma_{0}\right\}-2 \mu^{2}\left[\exp \left(-\sigma_{0} \eta\right)+1\right] \\
& -2{\sigma_{0}{ }^{2} .}_{C}(\Theta-1) / 2 \sigma_{0}\left(\mu^{2}-{\sigma_{0}}^{2}\right) \cosh (\mu)-\mu^{-1} \sinh (\mu) .
\end{aligned}
$$


Likewise, the boundary conditions are applied as $\Phi_{1}(\eta)=\Phi_{1}(1)$ for $\eta=1$, and $\Phi_{1}(\eta)=0$ for $\eta=0$, which yields the first order correction to the overall acceleration rate in terms of $\sigma_{0}$ :

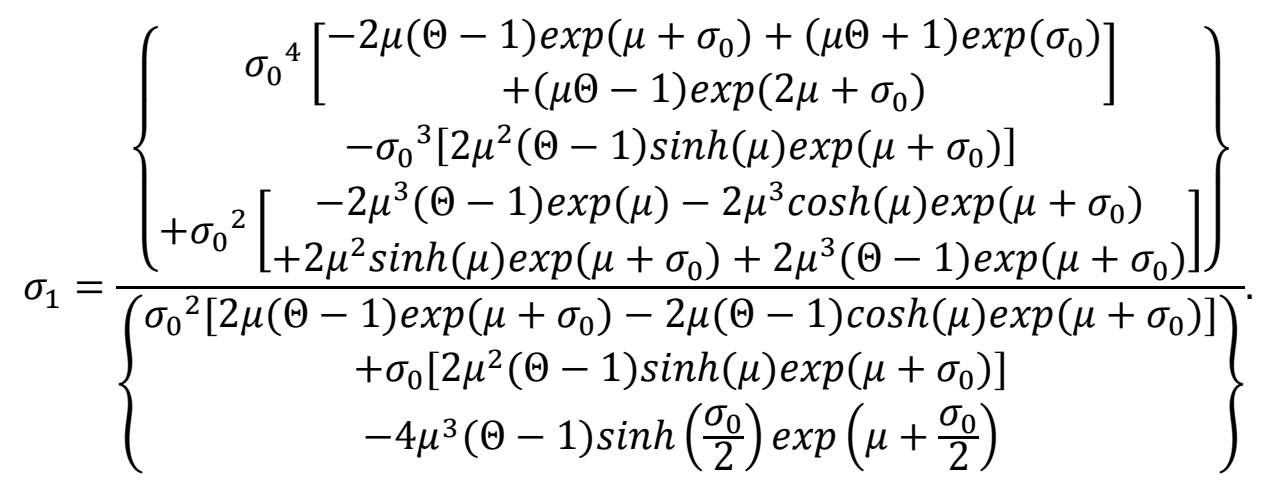

The last consideration would be about the fate of $\mu$ terms, which could be further simplified. It is observed that

$$
\mu=\sqrt{\sigma R e}=\sqrt{\left(\sigma_{0}+\epsilon \sigma_{1}\right) R e}=\sqrt{\left(1-\epsilon \frac{\sigma_{1}}{\sigma_{0}}\right) \sigma_{0} R e} .
$$

Now, let us check how $\epsilon \sigma_{1} / \sigma_{0}$ term compares with respect to the unity. Recalling the definition $\epsilon=\delta M k=M k L_{f} / R$, let us also consider the definitions for the Reynolds and Prandtl numbers, $R e=U_{f} R / v$ and $\operatorname{Pr}=v / D_{t h}$, respectively. Using these relations, one can obtain $\operatorname{Re}=$ $U_{f} R / \operatorname{Pr} D_{t h}$, or rearranging the terms, one gets:

$$
D_{t h} / U_{f} R=1 / \operatorname{Pr} R e
$$

Observe that, the flame thickness is defined as the ratio of thermal diffusion rate $D_{t h}$ and planar flame speed $U_{f}$, namely $L_{f}=D_{t h} / U_{f}$. Substituting this definition into Eq. (5.36), one obtains $L_{f} / R=1 / \operatorname{Pr} R e$. Therefore, the correction factor becomes

$$
\epsilon=M k / P r R e .
$$

Hence, using Eqs (5.31), (5.34), and (5.37), $\epsilon \sigma_{1} / \sigma_{0}$ terms can be calculated, whose trend could be seen in Fig. 5.7, and picked values are shown in Table 5.1. It is evident that these values become significantly small for $R e>10$, therefore it is plausible to use the approximation $\mu \cong \sqrt{\sigma_{0} R e}$, and substitute it into Eq. (5.34). Overall, the model equation for the total acceleration rate is hereby developed, which incorporates the internal flame structure as the first order correction term, recalled as follows: 


$$
\sigma=\sigma_{0}+\epsilon \sigma_{1}
$$

where the correction factor is given by Eq. (5.37).



Figure 5.6: Exponential flame acceleration rate $\sigma$ versus the Reynolds number for various fixed Lewis number values of simulation data. Same configuration of model equation (5.38) fitted in order to be compared to the simulation data.

Here, it is needed to introduce $M k_{\text {eff }}$ due to likewise reasons as discussed in Section 5.1. Hence, the correction factor is modified as

$$
\epsilon=M k_{e f f} / \operatorname{PrRe}
$$

where the $M k_{\text {eff }}$ is given by Eq. (5.13). An extensive plot of this model equation is shown in Fig. 5.6, where the simulation data is compared to the theory for various Le cases. The phenomenological constants of the effective Markstein number are likewise set to $\Gamma=0.001$ and $n=5.5$. Similarly, the theory provides qualitative agreement with numerical results, especially for higher $L e$ cases where flame thickening occurs. 


\begin{tabular}{|c|c|c|c|c|c|c|c|}
\hline $\boldsymbol{R} \boldsymbol{e}$ & 5 & 10 & 15 & 20 & 25 & 30 & 35 \\
\hline$\left|\boldsymbol{\epsilon} \boldsymbol{\sigma}_{1} / \boldsymbol{\sigma}_{\mathbf{0}}\right|$ & 0.317 & 0.121 & 0.068 & 0.045 & 0.032 & 0.025 & 0.020 \\
\hline
\end{tabular}

Table 5.1: Term $\epsilon \sigma_{1} / \sigma_{0}$ in Eq. (33) for varying Re number.



Figure 5.7: Term $\epsilon \sigma_{1} / \sigma_{0}$ versus Reynolds number. Apparent that the term could be conventionally neglected at least for $R e>10$. 


\section{Chapter 6: Summary and Conclusions}

This work hereby presents the analytical formulations and numerical simulation results of $M k-L e$ interplay effects on the flame acceleration scenario. Firstly, the coupling mechanisms in the Ze$L e-\Theta$ parametric space are investigated, where the threshold values are observed addressing the diffusive flame properties. Hence, the conditions for the flame stability for the $D T$ instability are defined in terms of the critical parametric space formed by $M k_{C}, Z e_{C}, E_{A, C}$, and $L e_{C}$ numbers. These results indicate that trough formation on the flame front due to the diffusive properties might be indeed the result of $D T$ instability, and dissipation of such trough effects in the long run as shown by simulations is verified via the $D T$ stability conditions, where the flame front is absolutely stable for given parameters.

While the previous numerical simulation on the topic was done only on the equidiffusive flames, i.e. $L e=1$, this work presents the direct numerical simulations of the hydrodynamic combustion equations including transport processes and chemical kinetics for non-equidiffusive flames $(L e \neq 1)$, in order to observe the effects of internal diffusive properties on the flame acceleration scenario in a deeper manner. First, the effects of $L e$ number on the flame acceleration are observed, with the acceleration rates for various Le-Re combinations compared to each other. The numerical simulation data are also compared to the previous theory and simulation results, and it is shown that a higher $L e$ number increases the flame thickness, hence reducing the flame acceleration in narrow tubes. Then, it is also demonstrated that $L e$ has unique effects on the morphology of flame front. Namely, if $L e$ is less then a critical value, $L e<L e_{C}$, flame propagation undergoes a variety of stages, which substantially increase the burning and acceleration rates through crest and trough formations. Simulations yielded enough evidence that such crest and trough formations disappear after some time in the $R e=10-35$ range, and the flame front once again acquires a globally convex shape until it triggers a DDT. Nonetheless, by undergoing the "trough instability" stages (at $L e<L e_{C}$ ), the flame acceleration is increased due to a larger flame surface area and total burning rate, since the morphological deformations yield bifurcation/channeling effects. Hence, it may facilitate a potential DDT scenario in quite shorter time periods. Overall, non-equidiffussive effects are promoted at lower $L e$, and larger channel width. 
On the other hand, the effects of internal flame structure on flame acceleration scenario are scrutinized from an analytical perspective. One may recall that previous analytic models were done with the Landau limit of zero flame thickness approximation. However, the corrective analytical formulae are hereby derived which incorporate internal flame structure parameters. The present new theory shows qualitative agreement with previous theory and simulation results [17], hence it is successful in describing the internal flame structure effects that appears at low $R e$ values. These corrections moderate fast otherwise (i.e. when $R e$ increases), which indicates that the internal flame structure effects do not change the qualitative scenario of the flame acceleration.

Moreover, the results of both numerical simulation data and theoretical predictions on the effects of $L e$ number on flame acceleration are combined. The theory is proved to be successful in describing the flame thickening effect which manifests itself as a reduction in flame acceleration at lower $R e$ values. Overall, despite quantitative differences, it is demonstrated that a qualitative harmony exists between computational and analytical results on the theory of nonequidiffusive flames.
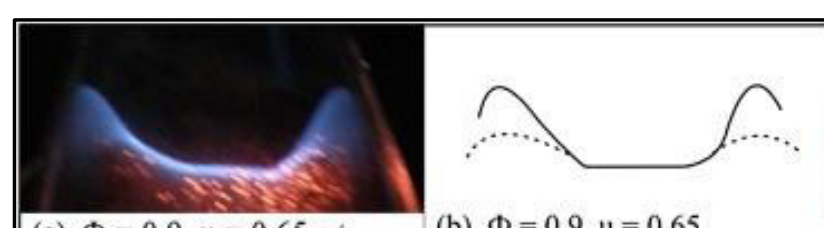

(b) $\Phi=0.9, \mathrm{u}=0.65$


(i) $\Phi=1.1, \mathrm{u}=0.23$

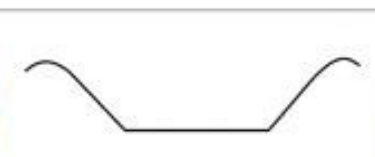

(f) $\Phi=1.3, \mathrm{u}=0.75$

(j) $\Phi=1.1, \mathrm{u}=0.23$

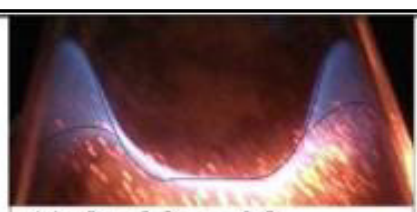

(c) $\Phi=0.8, u=0.9$

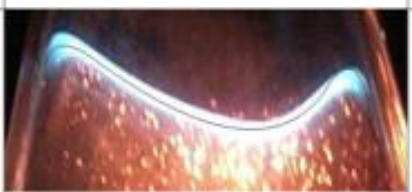

(g) $\Phi=1.2, \mathrm{u}=0.95$

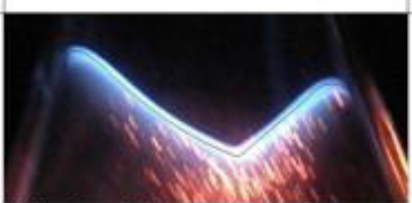

(d) $\Phi=0.8, \mathrm{u}=0.9$

(h) $\Phi=1.2, \mathrm{u}=0.95$
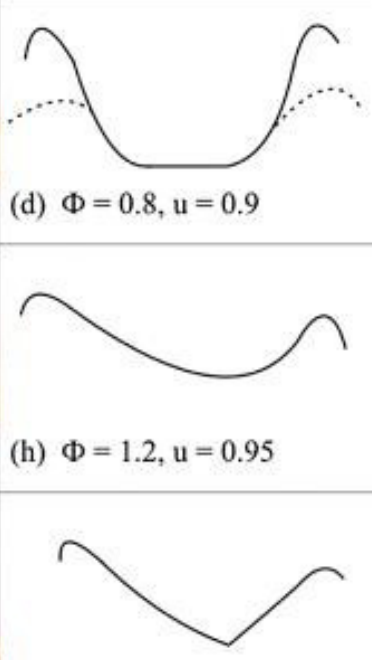

(l) $\Phi=1.1, \mathrm{u}=0.45$

Figure 6.1: Images of various flame propagation modes for equivalence ratio values $\Phi=0.8,0.9,1.1,1.2$, and 1.3, as well as flow velocity values $\mathrm{u}=0.23,0.45,0.65,0.75,0.90$, and $0.95 \mathrm{~m} / \mathrm{s}$. [49] 
A final remark would be on the possible practical applications of this study. Namely, the reader may ask how "non-equidiffusivity" or "finite-thickness" effects manifest themselves in experimental setups or nature. Certainly, there are various scenarios where one encounters nonequidiffusive combustion, especially when flow velocities and equivalence ratios are considered within a wide range. One such study has been conducted by Khandelwal and Kumar [49] for premixed methane-air mixtures, where the flow velocity and equivalence ratio effects on the flame morphology and dynamics were scrutinized. Figure (6.1) shows some pictures from their experiments for various cases. As the equivalence ratio is varied, the Lewis number of the premixed mixture changes, hence one observes similar morphological changes (e.g. channeling) as we described in this study.

Another similar study has been conducted by Bedat and Cheng [50], where they experimentally observed the morphology and dynamics of various fuel mixtures. The $\mathrm{OH}$ planar laser-induced fluorescence (PLIF) measurements taken for propane, methane, and hydrogen mixtures using a swirl burner are shown in Fig. (6.2). Each mixtures having different Lewis and Markstein numbers thus behave differently against the curvature and diffusional-thermal effects. Hence, the flamefront is observed to acquire a fractal cellular structure which might be stable or unstable under certain conditions depending on the Lewis number.

For further remarks on the coupled hydrodynamic and diffusional-thermal instabilities, the reader is encouraged to see the [51], where the effects of sub-unity Lewis numbers on flame morphology and stability are discussed further.
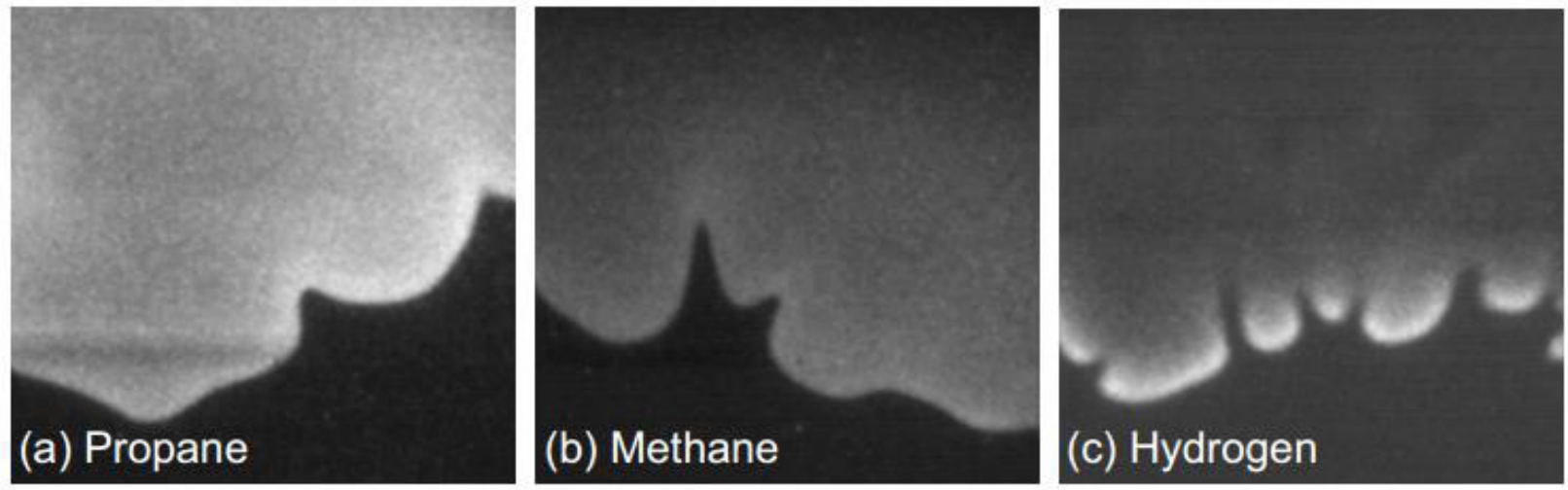

Figure 6.2: Experimental OH PLIF measurements for propane, methane, and hydrogen mixtures. The image widths correspond to $3 \mathrm{~cm}$. [50] 


\section{References}

[1] C.K. Law, Combustion Physics, Cambridge University Press, New York, 2006.

[2] W. Choi, S. Hong, J.T. Abrahamson, J.-H. Han, C. Song, N. Nair, S. Baik, M.S. Strano, Nature Materials 9 (2010) 423-329.

[3] Y. Ju and K. Maruta, Prog. En. Combust. Sci. 37 (2011) 669-715.

[4] S.R. Rockwell, A.S, Rangwala, Combust. Flame 160 (2013) 635-640

[5] G.D. Roy, S.M. Frolov, A.A. Borisov, D.W. Netzer, Prog. En. Combust. Sci. 30 (2004) 545.

[6] G. Ciccarelli, S. Dorofeev, Prog. En. Combust. Sci. 34 (4) (2008) 499-550.

[7] G. Cicarelli, C.T. Johansen, M. Parravani, Combust. Flame 157 (11) (2010) 2125-2136.

[8] S.B. Dorofeev, Proc. Combust. Inst. 33 (2011) 2161-2175.

[9] M. Kuznetsov, V. Alekseev, I. Matsukov, S. Dorofeev, Shock Waves 14 (2005) 205-215.

[10] M. Kuznetsov, M. Liberman, I. Matsukov, Combust. Sci. Technol. 182 (2010) 1628-1644.

[11] M. Wu, M. Burke, S. Son, R. Yetter, Proc. Combust. Inst. 31 (2007) 2429.

[12] M.-H. Wu, C.-Y. Wang, Proc. Combust. Inst. 33 (2011) 2287.

[13] M.-H. Wu,W.-C. Kuo, Combust. Flame 159 (3) (2012) 1366-1368.

[14] M.-H. Wu,W.-C. Kuo, Proc. Combust. Inst. 34 (2013) 2017-20124

[15] L. Kagan, G. Sivashinsky, Combust. Flame 134 (2003) 389.

[16] J.D. Ott, E.S. Oran, J.D. Anderson, AIAA Journal 41 (2003) 1391.

[17] V. Bychkov, A. Petchenko, V. Akkerman, L.-E. Eriksson, Phys. Rev. E, 72 (2005) 046307.

[18] V. Bychkov, D. Valiev, V. Akkerman, C.K. Law, Combust. Sci. Technol. 184 (2012) 1066.

[19] V. Akkerman, V. Bychkov, A. Petchenko, L.-E. Eriksson, Combust. Flame 145 (2006) 675.

[20] V. Akkerman, C.K. Law, V. Bychkov, L.-E. Eriksson, Phys. Fluids 22 (5) (2010) 053606.

[21] K. I. Shelkin, Zh. Eksp. Teor. Fiz., 10 (1940) 823.

[22] Ya. B. Zel'dovich, G. I. Barenblatt, V. B. Librovich, G. M. Makhviladze, Mathematical Theory of Combustion and Explosion, Consultants Bureau, New York, 1985.

[23] D. Valiev, V. Bychkov, V. Akkerman, L.-E. Eriksson, M. Marklund, Phys. Lett. A 372 (2728) (2008) 4850-4857.

[24] D. Valiev, V. Bychkov, V. Akkerman, L.-E. Eriksson, Phys. Rev. E 80 (2009) 036317.

[25] V. Bychkov, V. Akkerman, D. Valiev, C.K. Law, Phys. Rev. E 81 (2010) 026309.

[26] D. Valiev, V. Bychkov, V. Akkerman, L. Eriksson, C.K. Law, Phys. Fluids 25 (2013) 096101. 
[27] V. Bychkov and M. Liberman, Phys. Rep. 325, 115 (2000).

[28] G. I. Barenblatt, Ya. B. Zel'dovich, Prikl. Mat. Mekh., 21, No. 6 (1959) 856-859.

[29] Barenblatt et al 1962; ([10] G. I. Barenblatt, Ya. B. Zel'dovich, A. G. Istratov, Prikl. Mat. Tekh. Fiz., No. 4 (1962) 21-26.

[30] J. B. Rosen, Chem. Phys., 22, No. 4 (1954) 733-742.

[31] Ya. I. Kanel', Dokl. Akad. Nauk SSSR, 136, No.2, (1961) 277-280.

[32] A. M. Grishin, E. E. Zelenskii, in: Proc. $4^{\text {th }}$ Sci. Conf. on Mathematics and Mechanics (in Russian), Pt. 2, Tomsk (1974), pp. 674-675.

[33] B. Lewis, G. von Elbe, Combustion, Flames, and Explosion of Gases, New York, 1938.

[34] A. P. Aldushin, S. G. Kasparyan, Dokl. Akad. Nauk SSSR, 224, No. 1 (1979) 67-70.

[35] K. O. Sabdenov, Journal of Eng. Phys. And Thermophys., Vol. 75 (2002) No. 4.

[36] S. Kadowaki, T. Hasegawa, Prog. En. Combust. Sci. 31 (2005) 193.

[37] S. Kadowaki, H. Suzukia, H. Kobayashi, Proc. Combust. Inst. 30 (2005) 169-176.

[38] M. Matalon, B. J. Matkowski, J. Fluid Mech. 124 (1982) 239.

[39] V. Akkerman, V. Bychkov, Combust. Theory Modeling, 9 (2005) 323.

[40] G. Searby, J. Quinard, Combust. Flame 82 (1990) 298-311.

[41] S. Davis, J. Quinard, G. Searby, Combust. Flame 130 (2002) 123-136.

[42] R.W. Pitz, S. Hub, P. Wang, Prog. En. Combust. Sci. 42 (2014) 1-34.

[43] C. Hall, W. Kulatilaka, N. Jiang, J. Gord, R.Pitz, Proc. Combust. Inst. 35 (2015) 1107-1114.

[44] V. Bychkov, S. M. Golberg, M. A. Liberman, L. E. Eriksson, Phys. Rev. E 54 (1996) 3713.

[45] O. V. Travnikov, V. V. Bychkov, M. A. Liberman, Phys. Rev. E 61 (2000) 468.

[46] D. Valiev, V. Akkerman, M. Kuznetsov, L. Eriksson, C. Law, Combust. Flame 160 (2013) 97-111.

[47] A. Petchenko, V. Bychkov, V. Akkerman, L. Eriksson, Combust. Flame 149 (2007) 412.

[48] T. Poinsot, D. Veynante, Theoretical and Numerical Combustion, Edwards, Ann Arbor, 2005.

[49] B. Khandelwal, S. Kumar, App. Therm. Eng. 30, 17-18, 2718-2723 (2010).

[50] B. Bedat, R. K. Cheng, Combust. Flame 100 (1995) 485-494.

[51] J. Yuan, Y. Ju, C.K. Law, Phys. Fluids 17 (7) (2005) 074106. 